\title{
An Investigation into the Effect of Electrode Type and Stimulation Parameters on FES-induced Dynamic Movement in the Presence of Muscle Fatigue for a Voltage-Controlled Stimulator
}

\author{
Ashley M. Stewart, Christopher G. Pretty, XiaoQi Chen \\ Mechanical Engineering Department, University of Canterbury, \\ Christchurch, New Zealand
}

\begin{abstract}
Functional Electrical Stimulation (FES) is very useful in Stroke Rehabilitation for rebuilding muscle strength, both in a clinical setting and for home-based rehabilitation, however it is limited in its usage due to the resulting FES-induced muscle fatigue, as well as a large variation in muscle response. This paper compares the effectiveness of e-textile electrodes with conventional hydrogel electrodes at a range of stimulation parameters. The e-textiles produced a stronger response at a lower voltage than the hydrogels (3-4 V difference for a stimulation intensity voltage range of 8-22 V) and offer more consistency across different FES sessions. A simple model was then developed which relates the variation of the stimulation parameters (voltage, pulse-width, and frequency) to a change in angle for the elbow joint (Bicep muscle) using the e-textile electrodes. Step responses for various combinations of FES parameters were measured for 8 different healthy subjects for a total of fifty 10 minute long tests. Overall the model produced good predictions of the arm response and it was found that the model was more accurate and consistent for combinational parameter steps than for individual parameter steps. The $\mathrm{R}^{2}$ value for the measured step change compared with the predicted step change for FES-induced contractions for all step types and subjects combined was 0.76 for one of the methods using the model.
\end{abstract}

Keywords: Rehabilitation, home-based healthcare, functional electrical stimulation, hydrogel electrodes, e-textile electrodes

\section{INTRODUCTION}

Functional Electrical Stimulation (FES) is the application of electrical pulses to a muscle in order to induce functional movement [1]. It has many applications, with one of the key applications being its use in stroke rehabilitation to improve muscle strength. Muscles consist of hundreds to thousands of muscle fibres wrapped together in bundles surrounded by connective tissue. Each muscle fibre can be broken down into myofibrils which are further made up of stacks of sarcomeres consisting of actin and myosin filaments. Muscular contraction results when the actin and myosin filaments slide across one another. The sliding of the filaments is triggered when a motor neuron transmits an action potential. One action potential produces one contraction. A sustained contraction is the result of many action potentials in quick succession more specifically known as motor-unit action potentials (MUAPS). The motor neurons have a one to many relationship with the muscle fibres. Each muscle fibre is controlled by one motor neuron and each motor neuron controls several muscle fibres. There exist two main types of muscle fibres, slow-twitch and fast-twitch. The slow-twitch fibres are generally more fatigue resistant but also take longer to respond and produce less force. Fast-twitch fibres are faster to respond, capable of larger forces, but less fatigue resistant [2].

The force, and speed of the contraction depend on the spatial recruitment (the number of motor neurons transmitting) and on temporal recruitment (the frequency at which the action potentials are transmitted) [3]. During voluntary muscle contraction the muscle fibres recruited follow a specific order as observed by [4]. Smaller slow-twitch fibres have a lower activation threshold (respond to a lower spatial recruitment) and so are recruited 
prior to the larger fast-twitch fibres. That is, slow-twitch fibres respond slower but also earlier (at a lower recruitment) than the fast-twitch fibres. As a muscle contraction increases more of the fast-twitch fibres are recruited.

During functional electrical stimulation the fibres are recruited indiscriminately which contributes to an earlier onset of muscle fatigue as the more fatigable fast-twitch fibres are recruited more readily than they would be during a voluntary contraction [3]. Electrodes placed over the muscle belly stimulate all the motor units beneath them. The larger the stimulation amplitude, the larger the spatial recruitment as the current is able to penetrate deeper into the muscle and stimulate more motor units. The spatial recruitment of FES is affected by three key parameters; the voltage, the current, and the pulse-width.

Temporal recruitment during FES is affected by the frequency of the pulses. During voluntary contraction temporal recruitment is asynchronous whereas during FES-induced contractions temporal recruitment is synchronous. Modulation of temporal recruitment is also known as rate-coding and there have been some studies on isometric contractions (constant muscle length, varying force) in the lower limb that suggest that varying the temporal recruitment and spatial recruitment simultaneously during FES induced contractions may reduce FESinduced muscle fatigue [5-7]. Despite this, rate-coding is not used in clinical settings, there have been no studies on this strategy employed in the upper limb or which simultaneously vary all parameters controllable during FES (amplitude[current or voltage], pulse-width, and frequency), and the evidence towards combined spatial and temporal recruitment control as a FES-induced fatigue reduction tool is not yet conclusive [8].

A recent review was conducted into upper-limb hybrid exoskeletons [9] which highlighted the advantages hybrid exoskeletons (exoskeletons which combine FES with an actuator) have with regards to improving the precision of FES induced movements. However, little attention has been given towards optimising the control between the actuator and the FES with regards to reduction and management of FES-induced fatigue or with regards to adapting to user ability. FES control systems used for upper-limb hybrid exoskeletons simply ramp up stimulation intensity when fatigue is observed. FES control systems used outside of hybrid exoskeletons tend towards the other extreme where complex models requiring many measurements for model parameter estimates result in setup times which are unsuitable for clinical applications. Furthermore without the precision advantage from the actuator, non-hybrid FES systems generally place a larger focus on achieving precision rather than muscle fatigue reduction.

The design of a novel voltage controlled functional electrical stimulator has been described in other works [10] and is the FES device used during the tests described in this work. During initial testing of this device it was observed that the reusable Hydrogel electrodes commonly used during clinical stroke rehabilitation sessions involving FES, would degrade rapidly over several reapplications. Hydrogel electrodes are self-adhesive and consist of three layers; a top fabric layer, a conductive carbon layer, and a bottom hydrogel layer [11]. The hydrogel layer is predominately made up of water. The use of the term 'reusable' is only meant to indicate that the electrodes can be used more than once. They are however limited in the number of times they can be used. With reuse, the hydrogel electrodes will eventually deteriorate and dry out, losing their adhesiveness and effectiveness, and will need to be replaced.

This was an issue with regards to testing, as inconsistency with the electrodes contributes to inconsistency in muscle response. It was also difficult to ensure a consistent placement of these electrodes. In a hospital rehabilitation setting electrodes must also be replaced between different patients for hygiene reasons but the same electrodes are typically reused for a single patient until they become ineffective. Consistency of electrode placement within a hospital or home-based rehabilitation setting is difficult to achieve. Sometimes vivid is used to mark where to place the electrodes for the next session, however often the optimal electrode placement must be reassessed each session through trial and error.

In recent years e-textile electrodes have become the subject of much research for the purpose of human monitoring applications in the medical industry however few studies have been done that look at e-textiles for actuation purposes [12]. Two papers [13, 14] were found where textile electrodes were used for Transcutaneous Electrical 
Nerve Stimulation (TENS). TENS is very similar to Functional Electrical Stimulation (FES) however the goal in TENS is predominately that of pain management rather than actuation. Actuation may be induced during TENS however not to the same extent that it would be during FES.

The results from using e-textiles for TENS suggest that e-textiles could also be used for FES. This could have many benefits including: E-textile electrodes may last longer than the presently used hydrogel electrodes and because they are washable they may also be used for several patients rather than just one [14]. This has benefits in a research setting as well due to new electrodes not having to be purchased all the time and because a reduction in electrode degradation results in a more consistent response across several tests. The ability to sew the e-textile electrodes into clothing results in an improved portability and nicer look which will increase a patient's desire to use them. Furthermore it makes it possible to more consistently place the electrodes particularly with regards to the placement relative to one another. The global medical electrodes market is also forecasted to be a billion dollar industry by 2019 [15]. With aging populations the costs faced by healthcare providers can only be expected to increase. Increasing the reusability of stimulation electrodes could reduce some of these costs.

This paper uses the portable voltage controlled FES device described in previous work [10] to compare the performance of the e-textile electrodes with the conventional hydrogel electrodes. Tests will be conducted across a range of common stimulation parameters used for FES. Following the results from the electrode comparison, a new model is presented which relates all three controllable FES parameters (amplitude [voltage rather than current], pulse-width, and frequency) applied to the Bicep muscle to the rotation of the elbow joint in the sagittal plane for use in a hybrid exoskeleton. Several combinations of parameter steps are compared with regards to predictability and consistency for repetitive FES induced movements in the presence of muscle fatigue.

Section 2 describes the materials, set up and procedure for the comparison of the e-textiles and hydrogel electrodes. Section 3 presents the results and discussion for the electrode comparison tests. Section 4 introduces the novel model for the control of the FES. Section 5 describes the subjects and experimental procedure used for testing the FES model. Section 6 presents the results for the FES model tests. Section 7 discusses the results of the testing of the FES model, and finally Section 8 gives a brief summary and concluding remarks of the tests described in this paper.

\section{MATERIAL AND METHODS}

The e-textile electrodes used in this study have been made from commercially available conductive fabric [16]. The electrodes measure $50 \mathrm{~mm} \times 50 \mathrm{~mm}$ which is the same size as the commercial hydrogel electrodes produced by Verity Medical LTD which were used in these tests (Figure 1). The e-textile electrodes have a Velcro backing which attaches to the inside of an arm band which is wrapped around the upper arm over the bicep muscles. This ensures that the electrodes are in close contact with the skin and once the appropriate position is found the electrodes can be left in the same place for later testing resulting in a consistent positioning between testing and a fast setup time.

Tests were conducted over several days for no more than 2 hours a day. All tests were conducted on the right bicep. The duration of testing was limited each day to reduce any impact that muscle fatigue may have on the results. All of the tests were conducted on one healthy individual in 4 groupings each day with test groupings 1 and 4 using the hydrogel electrodes and test groupings 2 and 3 using the e-textile electrodes. Within each grouping every value tested for the varied parameter was applied at least twice in random order. The one exception is the ramp rate tests which were conducted over two days with different electrodes used each day. 


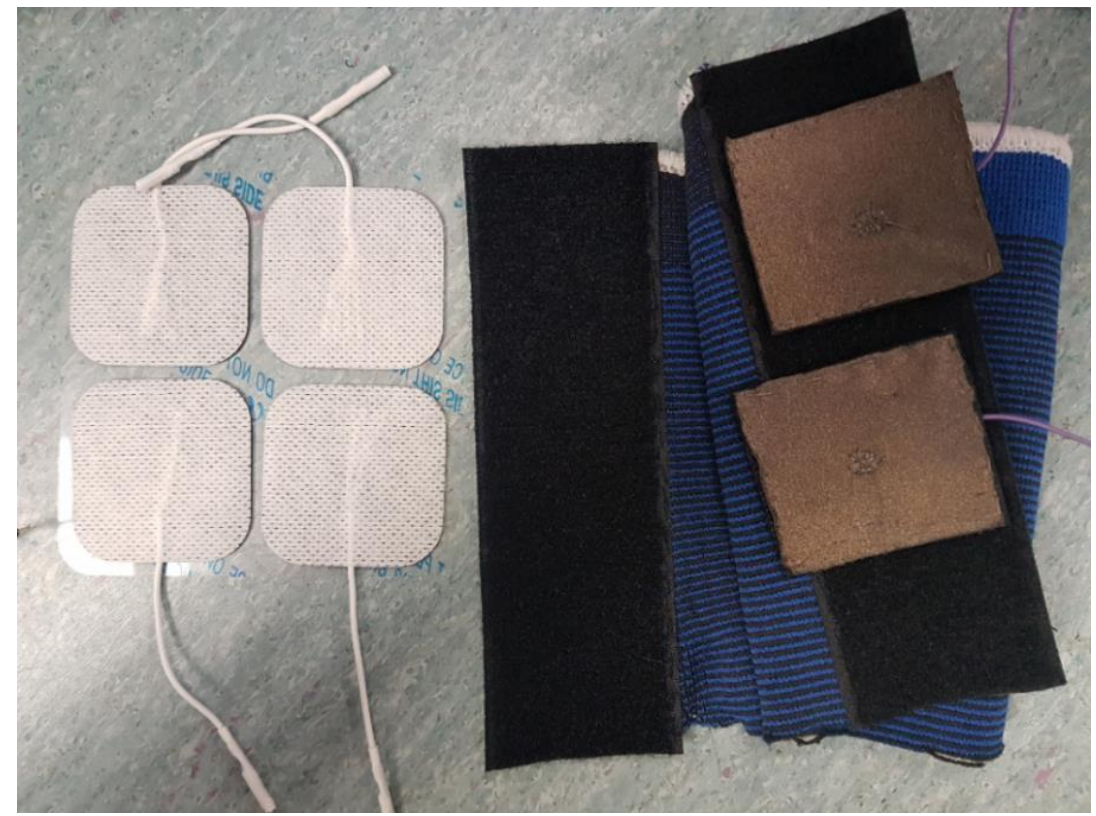

Figure 1: Hydrogel Electrodes (left) in Comparison with the E-textile Electrodes (right)

Measurement of the bend angle of the arm was accomplished using a commercially available Flex Sensor 4.5" [17] which is attached to an arm band and fitted over the elbow. Only the very end of the Flex Sensor is fixed (using adhesive), the rest of the sensor is held in place with sewing thread which allows the sensor to bend with the elbow. The Flex Sensor is shown in Figure 2. To check calibration of the Flex Sensor photos were taken of the arm at selected angles and the angle measured in the photos was compared with the angle calculated from the measured resistance. These values were found to be reasonably consistent and linear. Prior to each test, the subject was seated on a chair and the arm was held straight (relaxed), and pointing towards the floor. The Flex Sensor offset voltage was taken at this position. This angle is defined as $0^{\circ}$ and physical measurements were consistant with this value. Then the elbow is bent to a relaxed maximum bend. This voltage is measured and the angle is defined to be $120^{\circ}$ based on a measurement taken prior to commencement of testing. This improves the consistancy of the measurement of the arm angle between tests as it accounts for any sensor position shift between tests. However this method relies on a consistant bend of the arm when the maximum angle measurement is taken prior to each test and experiments showed this could be underestimated by up to $10^{\circ}$.

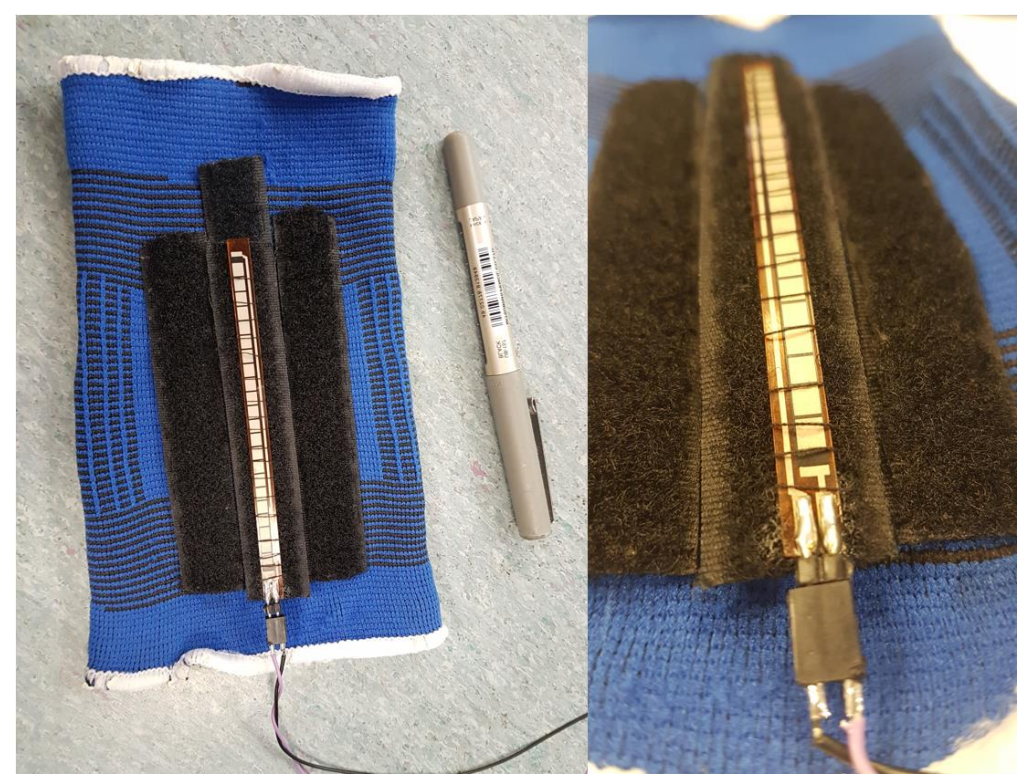

Figure 2: Flex Sensor 4.5” 
In all electrode comparison tests unless otherwise stated the frequency, pulse-width, ramp rate and stimulation duration were all arbitrarily and respectively predefined as; $30.5 \mathrm{~Hz}, 600 \mu \mathrm{s}, 10 \mathrm{~V} / \mathrm{s}$, and $10 \mathrm{~s}$. The ramp rate tests were the first tests conducted and it was observed from the results of these tests that $30 \mathrm{~s}$ was too long for stimulation application as the response of the muscle greatly decreased after about $20 \mathrm{~s}$. It was observed that $10 \mathrm{~s}$ was sufficient time to measure the peak response and a lower stimulation duration is preferable as it reduces the rate of FES induced muscle fatigue. Thus all tests with the exception of the ramp rate tests were conducted at $10 \mathrm{~s}$ stimulation duration.

Prior to the beginning of testing each day a manual test would be conducted with both electrode types to check the maximum comfortable voltage and ensure correct electrode placement. The ramp rate for the manual tests was varied and controlled by the operator. The frequency and pulse-width were constant and predefined as $30.5 \mathrm{~Hz}$ and $600 \mu$ s respectively. After these manual tests the test mode would be switched to automatic where the maximum amplitude, ramp rate, amplitude, frequency, pulse-width, and stimulation duration are all predefined. In this mode stimulation is initiated when the potentiometer is rotated beyond a threshold by an operator. The stimulation was then applied for the specified duration or until the potentiometer is turned below the threshold, whichever occurs first. This ensures that the stimulation can be stopped at any time. All tests are conducted using biphasic pulses as seen in Figure 3.

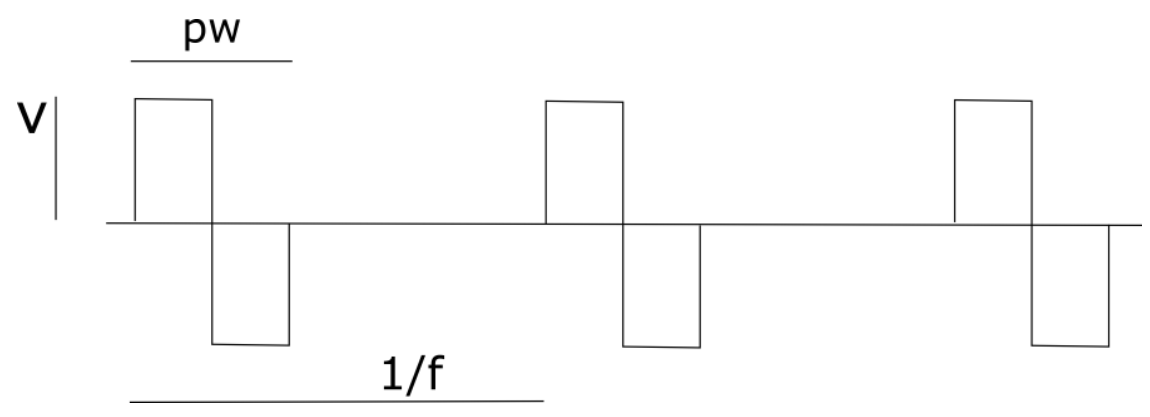

Figure 3: Biphasic Pulses

The maximum amplitude selected in each test, with the exception of the Amplitude tests, for a paticular electrode was the amplitude which could visually produce an approximate $80^{\circ}$ bend during the manual test. Thus, the maximum voltage is often different for the different electrodes. The reasoning for this is if the maximum voltage was too low it would be difficult to get a good muscle response and if the maximum voltage was too high then the stimulation can become too uncomfortable. In addition, stimulating the muscle at too high an amplitude can affect the recruitment order of motor units and increase the rate of fatigue [18]. To have the same maximum voltage used for both types of electrodes resulted in either the hydrogel electrodes not producing an adequate response, or the e-textile electrodes becoming uncomfortable.

All tests conducted on the e-textile electrodes described in this section were conducted using wet electrodes. The e-textile electrodes were wetted with tap water. Conductive gels, such as ultrasound gel could be used instead and produce a similar effect. Once the e-textile electrodes dry out the stimulation response can be reduced as the resistance increases. As the e-textile electrodes dry out, they can also become less comfortable to use due to the non-uniform wetness resulting in an uneven charge distribution. However, once wetted the e-textile electrodes will stay sufficiently wet for 2-3 hours so for short-term use this is not an issue. Dry e-textile electrodes can still produce a desirable response if their contact against the skin has sufficient pressure. The band used for the electrode comparison tests (Figure 1) did not provide enough pressure to produce a response in this subject without the conductive medium (water or gel). An arm band developed following the electrode tests but prior to the tests described in Section 5, was able to apply more pressure and could produce a response in this subject without the conductive medium although the response was typically more comfortable when a conductive medium was used. FES devices may be voltage or current controlled. Current controlled devices produce a more consistent response, as their performance is not affected by variance in skin impedance, however they often require higher voltages and are more complex and expensive to design $[19,20]$. The FES device used in these tests is a voltage controlled device as described in [10]. 


\section{COMPARISON OF ELECTRODES}

The results for the electrode tests are described in five subsections. Section 3.1 describes the effect on the muscle response for variation in the maximum amplitude of the stimulation. Section 3.2 evaluates the effect of different stimulation ramp rate. Section 3.3 presents results on the effect of varying the stimulation frequency. Section 3.4 investigates the effect of stimulation pulse-width variation. Section 3.5 discusses the overall results of the electrode comparison tests.

\subsection{Amplitude}

These tests were conducted with a ramp rate of $10 \mathrm{~V} / \mathrm{s}$, a frequency of $30.5 \mathrm{~Hz}$, a pulse-width of $600 \mu \mathrm{s}$, and a total duration of $10 \mathrm{~s}$. A range of voltages were tested ranging from the minimum voltage required to produce movement of the arm up to the minimum voltage required to achieve a full arm bend (approximately $120^{\circ}$ ). Seven different amplitudes, ranging from $8 \mathrm{~V}$ up to $22 \mathrm{~V}$, were tested for each electrode. The results can be seen in Figure 4 . The measured amplitude applied to the electrodes differs and will vary slightly in comparison with the desired amplitude due to variation in impedance at the electrode-skin junction. Variation within each test is minimal and always less than $0.25 \mathrm{~V}$. The results recorded for each test are the measured voltages, so variation across tests does not affect the results. Typically, the measured voltage variation between tests is also small at less than $1 \mathrm{~V}$.

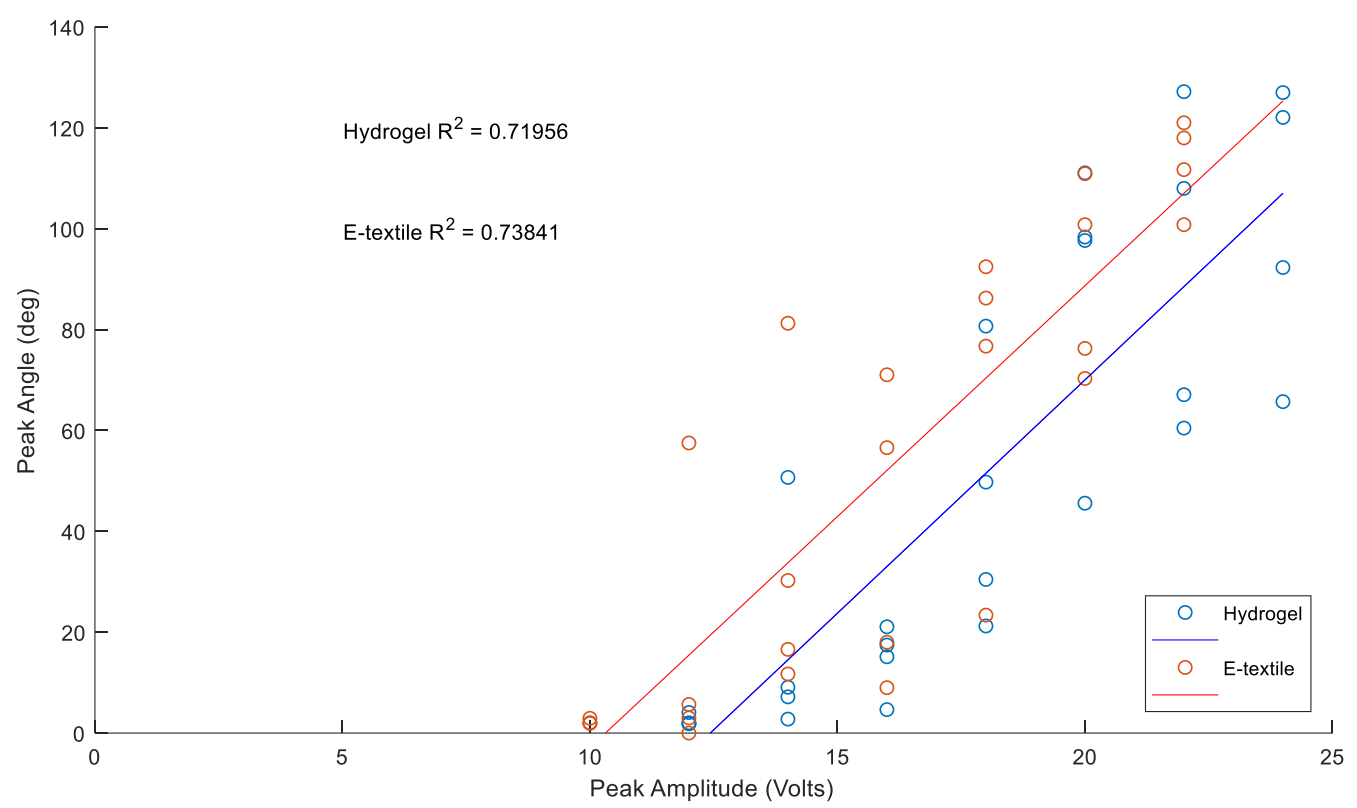

Figure 4: Effect of stimulation amplitude on peak arm bend

It was observed that the muscle response would typically be very small until a threshold voltage was reached. Once this threshold voltage was reached small changes in the voltage resulted in a much larger muscle response. Figure 5 to Figure 7 display the response of the arm in three of the different amplitude tests. In general, the etextiles required a lower voltage to achieve the same movement as the hydrogels by approximately $3-4 \mathrm{~V}$. At a similar voltage there was about a $20^{\circ}$ difference in arm bend between the two types of electrodes.

As the stimulation amplitude increased, the size of the contraction also increased. This is consistent with results reported in literature [21]. Another observation that can be made is that the arm reaches its peak angle at the very beginning of the delivery of maximum amplitude. As the amplitude is held constant the size of the muscle contraction will slowly decrease over time. These observations can be made for both types of electrodes. 


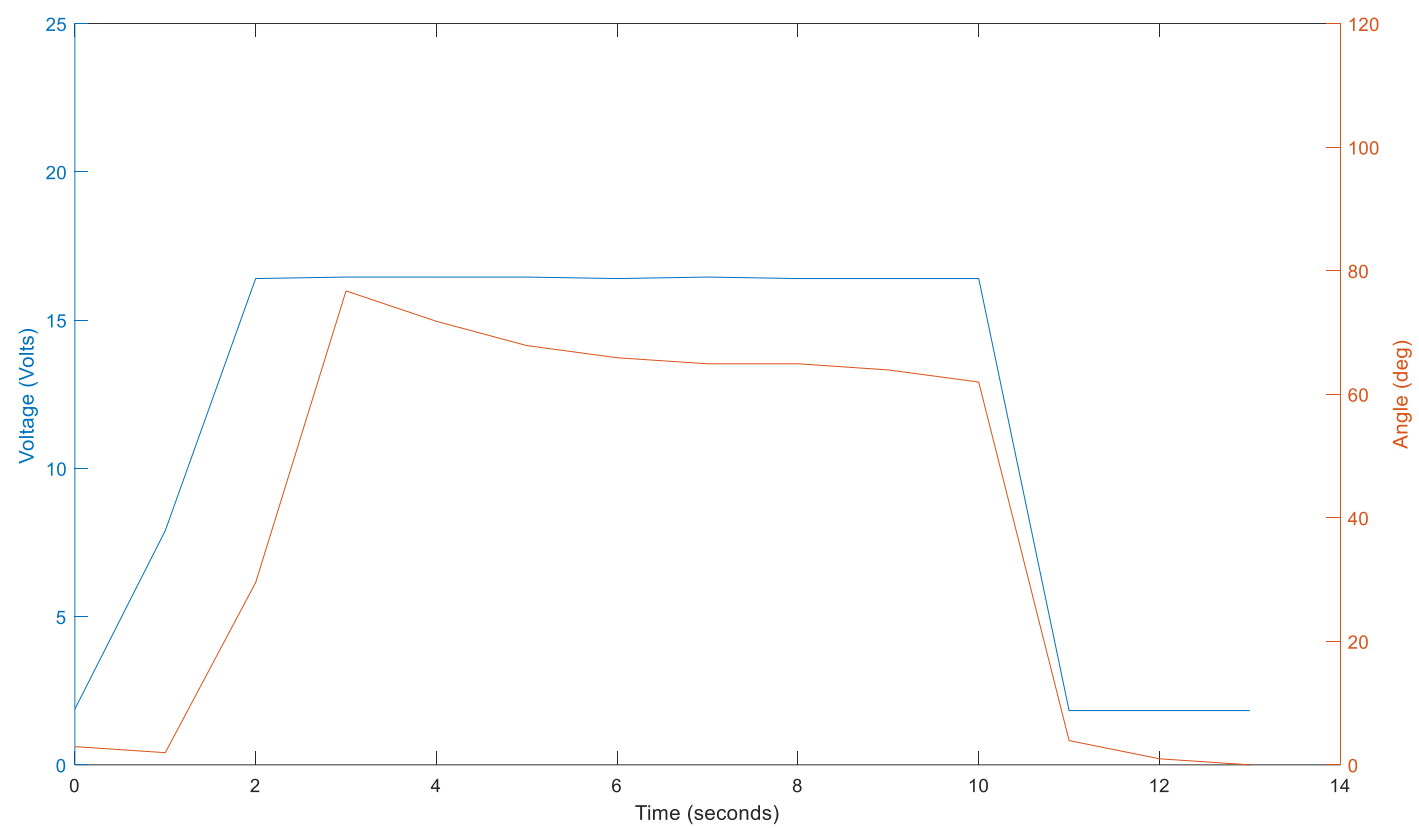

Figure 5: Arm response to $16.5 \mathrm{~V}$ stimulation using e-textile electrodes

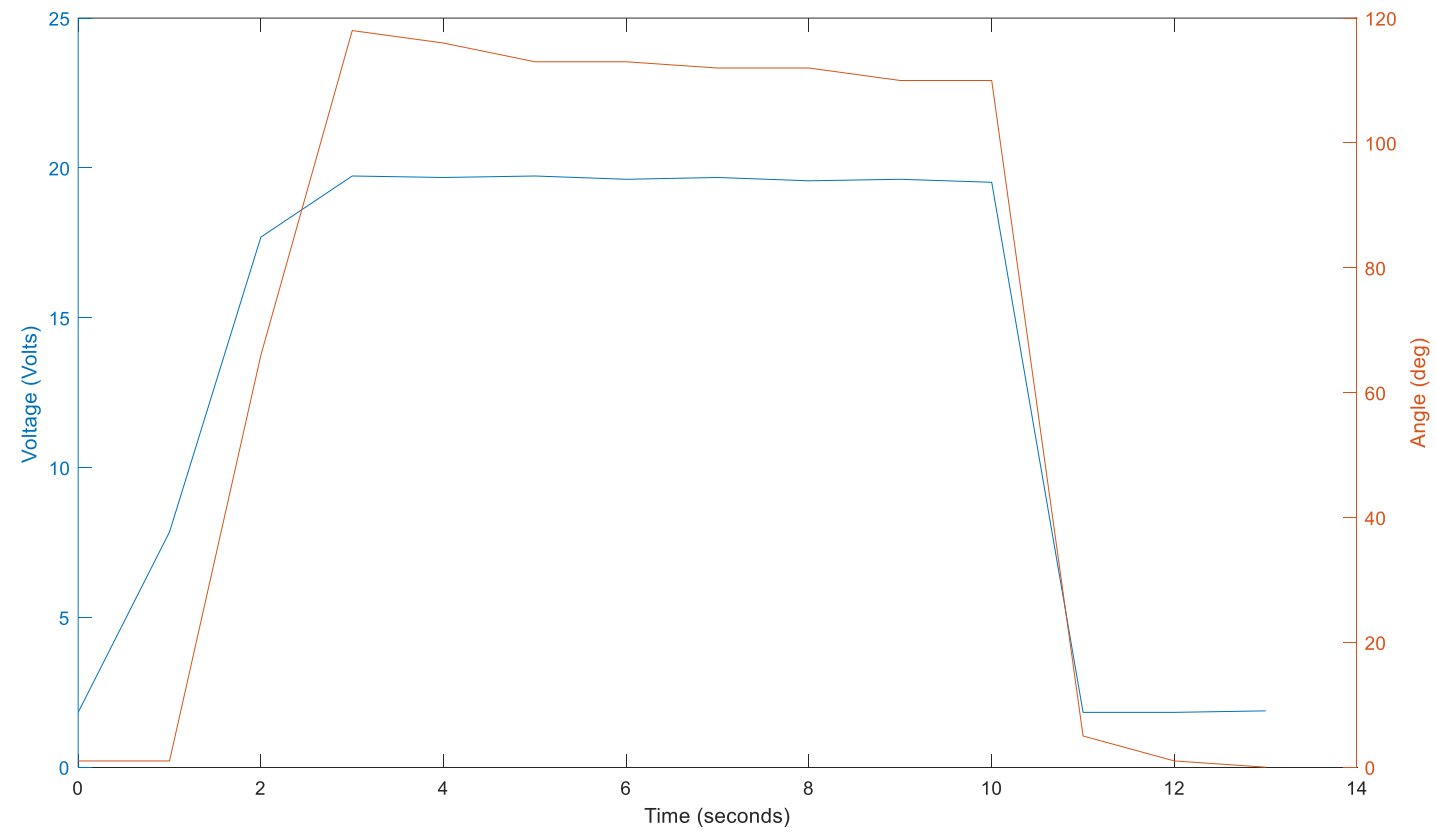

Figure 6: Arm response to $20 \mathrm{~V}$ stimulation using e-textile electrodes 


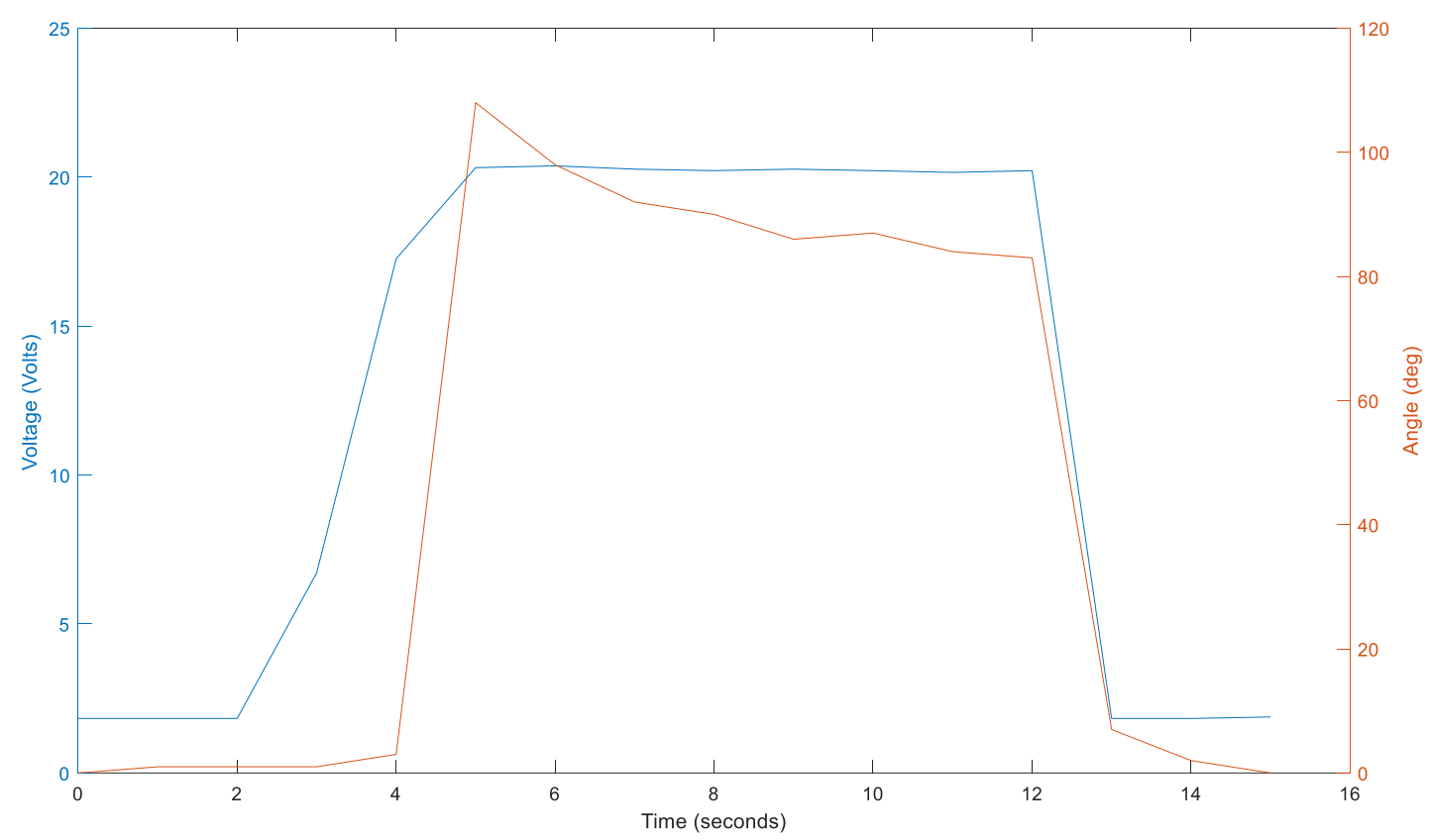

Figure 7: Arm response to $20 \mathrm{~V}$ stimulation using hydrogel electrodes

\subsection{Ramp Rate}

The ramp up rate is the rate at which the stimulation intensity approaches its maximum amplitude (Figure 8). This is not to be confused with the rise rate which is the rate at which an individual pulse approaches its maximum amplitude. Stimulation may be ramped up and/or ramped down. Longer ramp up times are sometimes used for patients with hypertonic or spastic muscles or for patients with a high sensitivity to the stimulation [21]. Ramp down is most often used to produce a more smoothly controlled movement.

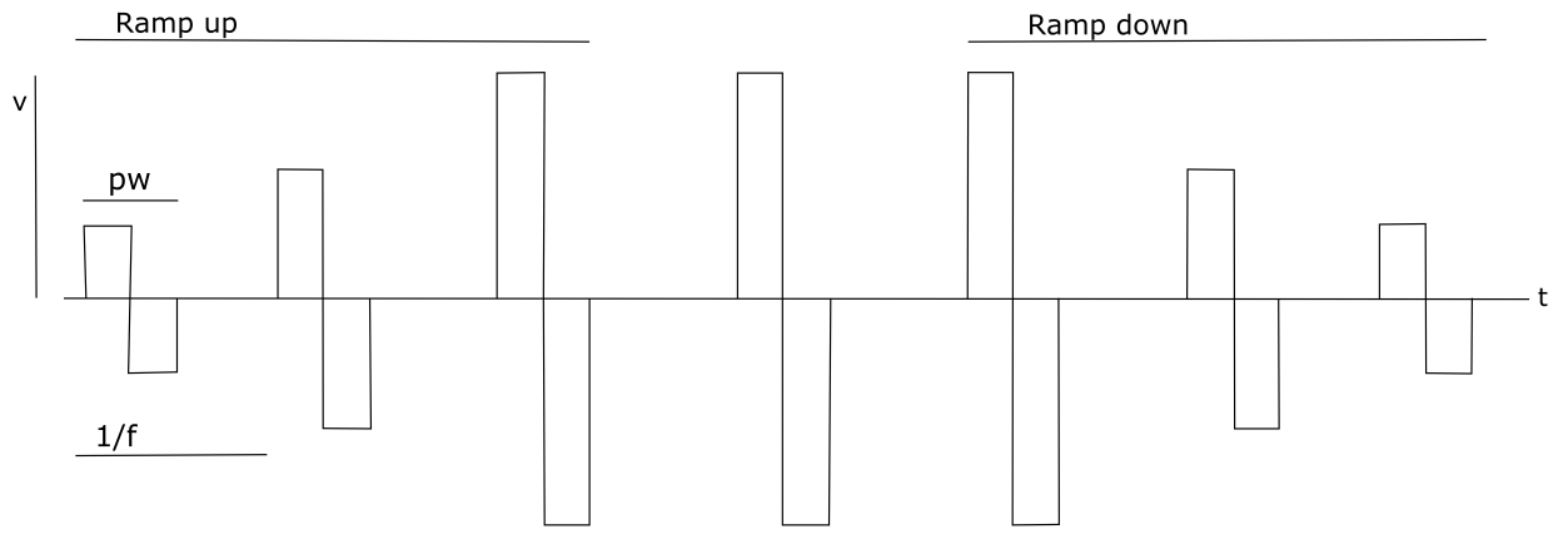

Figure 8: An example of ramped stimulation

Several tests were conducted using different ramp rates ranging from $2 \mathrm{~V} / \mathrm{s}$ up to $10 \mathrm{~V} / \mathrm{s}$. Stimulation was applied in each test for a duration of $30 \mathrm{~s}$. The frequency was fixed at $30.5 \mathrm{~Hz}$, and the pulse-width was fixed at $600 \mu \mathrm{s}$. The measured peak amplitude used was $18 \mathrm{~V}$ for the e-textile electrodes and $22 \mathrm{~V}$ for the hydrogel electrodes. It was observed that a faster ramp rate resulted in larger contractions and the response to variation in ramp rate was similar for both types of electrodes as can be seen in Figure 9. The e-textiles appear to be slightly less affected by variation in ramp rate than the hydrogels. However, the difference is not clinically significant. 


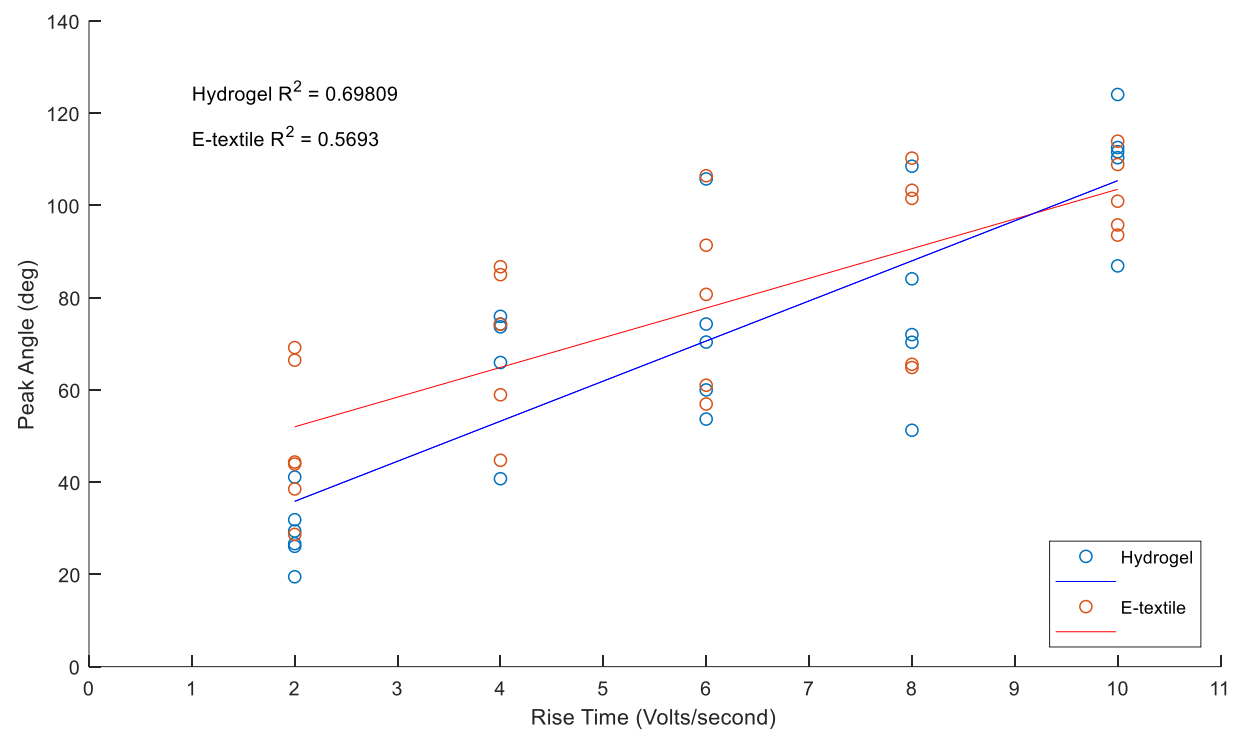

Figure 9: Effect of stimulation ramp rates on peak arm bend

\subsection{Frequency}

The frequency is the number of pulses per second. Typically FES devices deliver pulses at a constant frequency between $20-50 \mathrm{~Hz}$ although sometimes higher frequencies $(>80 \mathrm{~Hz})$ are also used [3]. Higher frequencies have been shown to increase the rate of FES induced fatigue [21]. Higher frequencies are often reported to be more comfortable and this was observed during these tests. Several tests were conducted at different frequencies ranging from $30.5 \mathrm{~Hz}$ up to $70 \mathrm{~Hz}$. Stimulation was applied in each test for a duration of $10 \mathrm{~s}$. The pulse-width was fixed at $600 \mu \mathrm{s}$, and the ramp rate was fixed at $10 \mathrm{~V} / \mathrm{s}$. The measured peak amplitude used was $18 \mathrm{~V}$ for the e-textile electrodes and $20.5 \mathrm{~V}$ for the hydrogel electrodes.

It is well documented that higher frequencies will produce stronger contractions [22, 23] and the results produced here are consistent with literature. Both types of electrodes produced a similar response to variation in frequency, as can be seen in Figure 10. The e-textiles appear to be slightly less affected by parameter variation than the hydrogels however, the difference between the electrodes for frequency variation is not clinically significant.

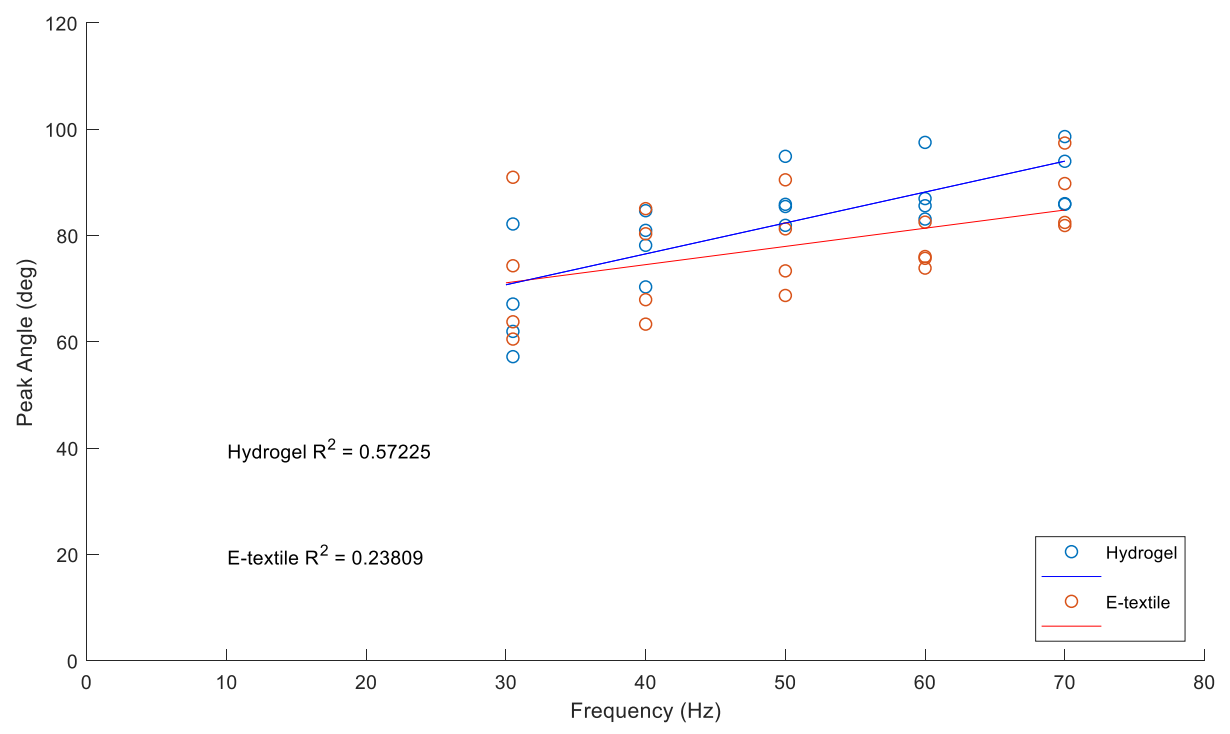

Figure 10: Effect of stimulation frequency on peak arm bend 


\subsection{Pulse-width}

The pulse-width is the length of the entire pulse. For a biphasic pulse this includes both the positive and negative phases [21]. Pulse-widths used by FES devices can range up to $1600 \mu \mathrm{s}$ with most devices using pulse-widths in the range of 100-500 $\mu$ s [24]. Larger pulse-widths will penetrate deeper into the subcutaneous tissue. Several tests were conducted using different pulse-widths ranging from $100 \mu \mathrm{s}$ up to $1000 \mu \mathrm{s}$. Stimulation was applied in each test for a duration of $10 \mathrm{~s}$. The frequency was fixed at $30.5 \mathrm{~Hz}$, and the ramp rate was fixed at $10 \mathrm{~V} / \mathrm{s}$. The measured peak amplitude used was $14 \mathrm{~V}$ for the e-textile electrodes and $20 \mathrm{~V}$ for the hydrogel electrodes. Results are displayed in Figure 11.

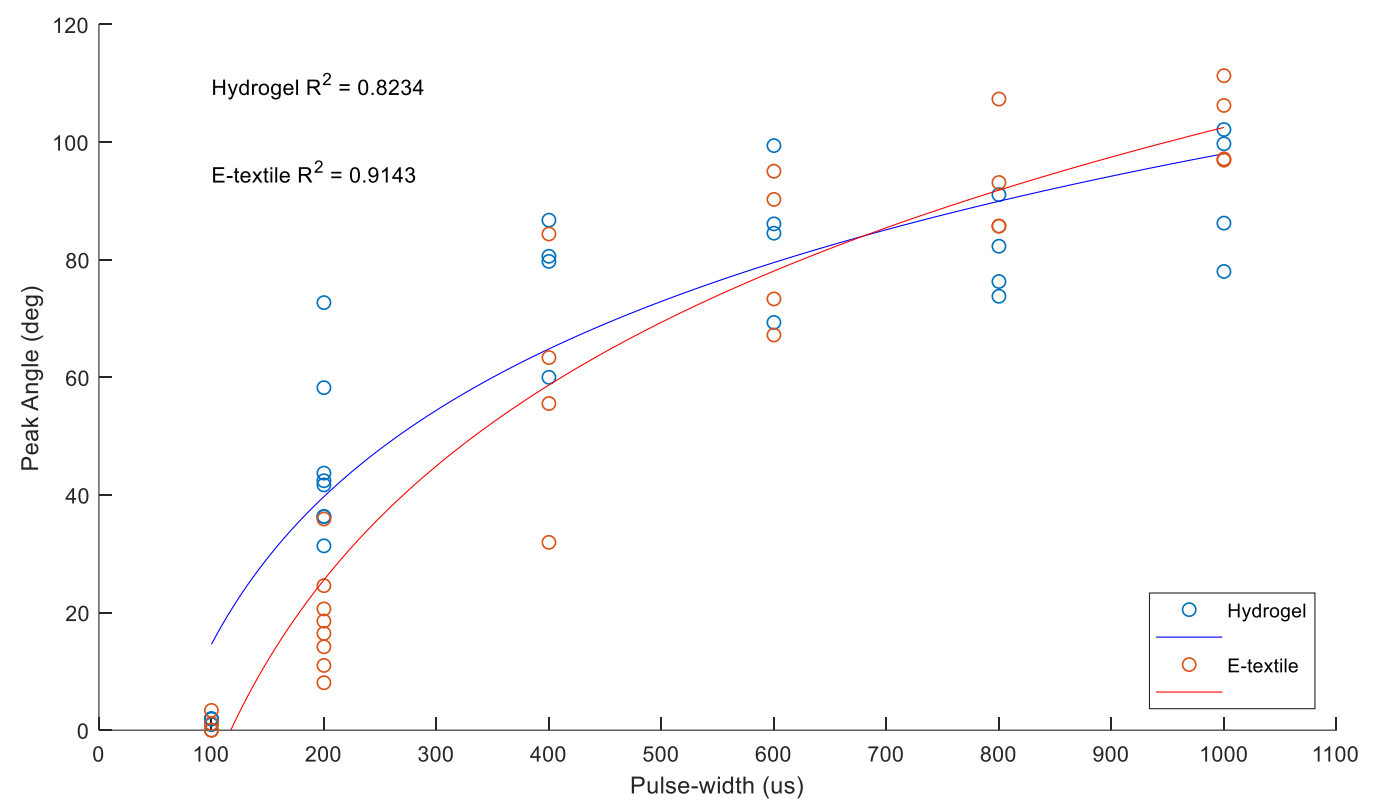

Figure 11: Effect of stimulation pulse width on peak arm bend

Despite the general consensus that the relationship between pulse-width and FES-induced torque is logarithmic there are many FES control schemes that treat the relationship as linear to reduce complexity [7, 25]. For comparison Figure 12 shows the results for the pulse-width tests with a linear fit instead of a logarithmic fit. While the logarithmic fit is obviously better, there is not a huge difference between the two models, especially for the etextile electrodes, such that a linear approximation for the purpose of controlling the pulse-with may prove good enough, particularly in the context of hybrid exoskeletons where precision is achieved through the use of the electromechanical actuator rather than the FES. 


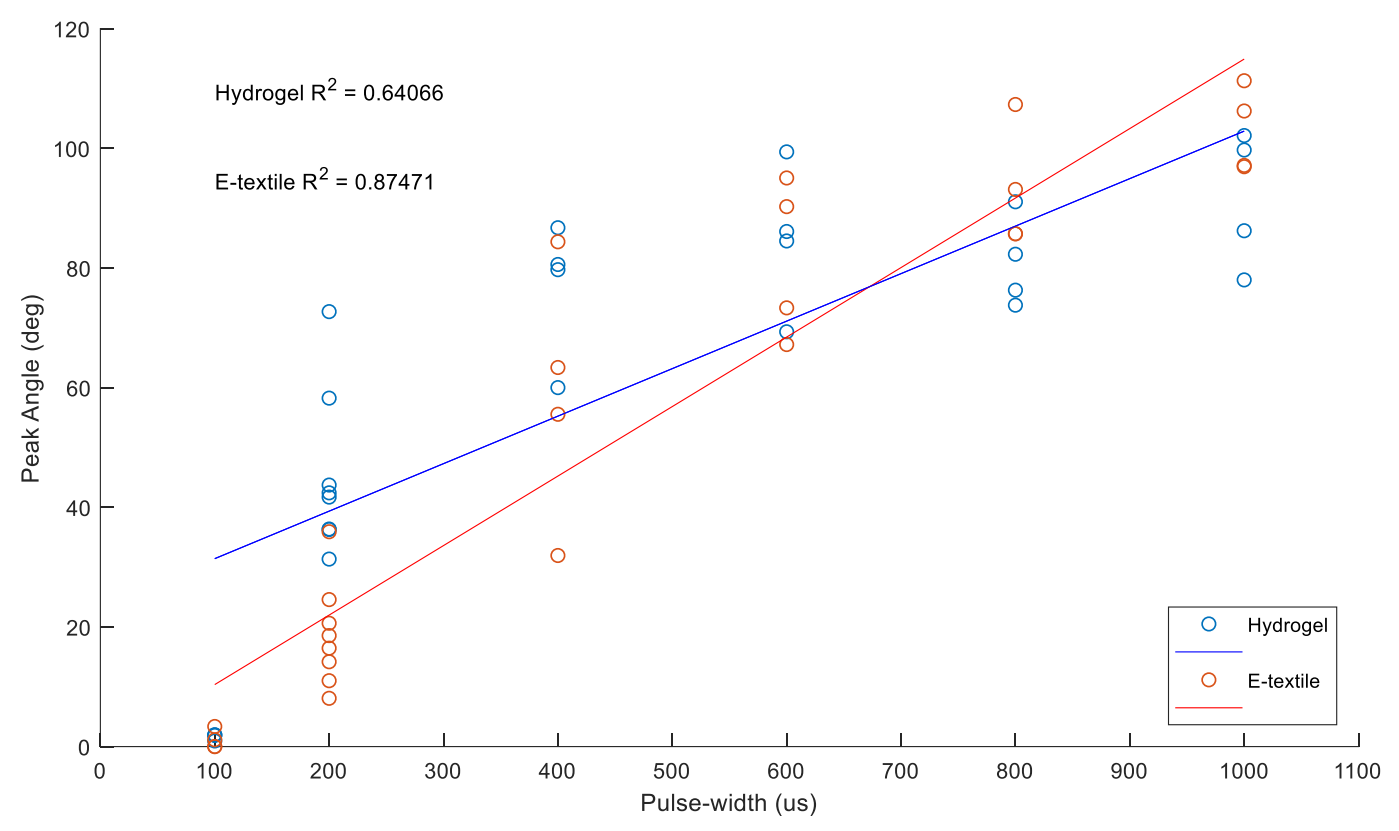

Figure 12: Effect of stimulation pulse width on peak arm bend with linear fit

Larger pulse-widths have been shown to increase the size of a contraction up to a point [21]. From about $200 \mu \mathrm{s}$ and below, a decrease in pulse-width will significantly decrease the size of the muscle contraction and increase the stimulation intensity required to produce movement [22]. Above $500 \mu \mathrm{s}$, variation in pulse-width will not significantly affect the muscle response. This was observed during these tests. What is interesting to note is that the e-textile electrodes were more strongly affected by variation in pulse-width than the hydrogel electrodes. The induced muscle response of stimulation with the e-textile electrodes decreased at a much faster rate below $400 \mu \mathrm{s}$ than it did for the hydrogel electrodes. Above $400 \mu$ s the stimulation muscle response from the e-textiles increased with increasing pulse-width at a faster rate than for the hydrogel electrodes.

\subsection{Summary}

These tests demonstrate the effectiveness of e-textile electrodes for FES applications. The e-textile electrodes have many advantages over the hydrogel electrodes. Both electrodes are capable of producing a contraction in the bicep muscle resulting in full flexion of the elbow joint. Previous studies have shown e-textiles to have a low impedance which is desirable for FES [26, 27]. This lower impedance results in the lower stimulation intensity required for muscle activation which is evident in these results. The lower required stimulation intensity has advantages such as lower power consumption of the FES device and improved safety.

The responses of the tested electrodes to variation in frequency and ramp rate were very similar and consistent with previous studies [21]. The differences seen in the electrode responses to variation in pulse-width are possibly a result of the e-textile electrodes having a higher capacitance than the hydrogels [27]. For stimulation applications requiring shorter pulse-widths $(<300 \mu \mathrm{s})$ the hydrogel electrodes may be preferable. There was significant variation between individual tests of the same electrode. In some cases for tests done on the same electrode with the same parameters, up to a $60^{\circ}$ difference in arm angle was observed. There are a few possible reasons for this. One is the method used for measurement of the angle, which could be underestimated in some cases by up to $10^{\circ}$. Another source of error is variation in placement of electrodes. While utmost care was taken to place the electrodes in the same location over the muscle it is possible that some shift occurred between test groups. Different conditions of the muscle could also result in the variation seen (e.g. muscle fatigue).

Over the test period the hydrogel electrodes had to be replaced three times, prior to the beginning of a test, due to deterioration in their stimulation response and a reduction in stickiness. In comparison, the response of the e-textile electrodes did not change even after they underwent one wash in a regular household washing machine. The 
longevity of the e-textiles is a significant advantage and could save medical providers considerable costs. In addition, the consistency in performance gives the e-textile electrodes an advantage in a research setting as results in studies which use e-textile electrodes will not be affected by electrode deterioration to the same extent as they would be if hydrogel electrodes were used without regular replacement.

There are some downsides to the use of the e-textile electrodes. One is that the e-textiles performance and comfort is significantly improved with the use of a conductive medium such as water or conductive gel. This limits their application to short sessions. For a typical rehabilitation setting lasting an hour or for research purposes this is not much of an issue. However, it may make e-textile electrodes unsuitable for applications such as aiding a person throughout the day with activities of daily living. Some investigation has been done into maintaining the wetness of the e-textile electrodes for use in electrocardiography so this could be less of a barrier in future [28]. The selfadhesiveness of the hydrogels offer one advantage over the e-textile electrodes. However, the ability to embed e-textiles into clothing thus improving visual appeal and consistency of electrode placement may make up for this as the most important factor in determining which electrodes are better for use in FES is whether the patient will use them. If a patient does not want to use the electrodes for any reason then it does not matter what other benefits they may have.

Comfort of the electrodes is an important feature to consider. Previous research suggests that e-textiles are as comfortable as conventional hydrogel electrodes and that they can reduce skin irritation for long term use [27]. During the tests described in this paper, it was observed that the hydrogel electrodes were slightly more comfortable than the e-textile electrodes, especially at lower frequencies. However, as these tests were only conducted on one individual few conclusions can be made about the comfort of the electrodes from these tests. In addition, the discomfort of stimulation from either electrode type was not observed to be any greater than the discomfort of removing the sticky hydrogel electrodes from the subject's skin. As stimulation discomfort is something that is a known limitation for FES usage, investigation into electrodes which can reduce discomfort is something that should be the focus of future research.

Overall, the e-textile had a similar performance to conventional hydrogel electrodes over the range of different stimulation parameters used in FES. E-textile electrodes offer many advantages for FES applications such as longevity and a lower impedance which could result in saved costs for healthcare providers and improved safety. However, their uptake may be limited by their comfort and requirement that they remain wet. Due to their reusability and improved consistency all tests described in Section 4 to 6 of this work use the e-textile electrodes in conjunction with conductive gel (Spectra 360).

\section{THEORY OF FES INDUCED MUSCLE MOVEMENT}

Control of FES can be divided into two main areas, control of precise movement and control of fatigue. Control of precise movement with FES is very difficult as the muscle response to FES is time-varying, and non-linear [29]. Muscle models are often used for control of FES-induced movements, with the most commonly used one being the Hill-muscle model and variations [30, 31]. However even the simplest implementations of these muscle models involve several parameters which are difficult and/or time-consuming to estimate and often require measurement of the maximum voluntary contraction (MVC) of the subject, something which is not possible in someone with no ability to move and which is difficult to achieve practically even in healthy subjects [32]. Stroke patients often suffer from complete paresis of the limb during the early days of recovery making MVC measurements impossible.

There are several other issues that arise when trying to produce precise muscle movements with FES. A single muscle sometimes controls several different movements so it's possible for stimulation to cause movements in addition to those intended. For example, simulating the bicep can sometimes produce unwanted rotation of the wrist. With surface electrodes it is also possible to stimulate more than one muscle simultaneously. Use of nerve cuff electrodes can alleviate some of these issues however nerve cuff electrodes require invasive surgery. Some investigation has been done into electrode arrays which may prove promising [33, 34]. The use of hybrid exoskeletons, which are the focus of this work have already been shown capable of improving FES precision [9] 
thus the main goal with regards to FES for this work is towards reducing the FES-induced fatigue. It is also in this area that the most gaps remain [35].

A model with a short setup time is of highest priority, as discussion with physiotherapists involved in stroke rehabilitation at Burwoood Hospital in Christchurch, New Zealand, emphasized that any system which took longer than present tools to set up would not likely be useful, even if it improved rehabilitation measures and/or outcomes. Simplicity is preferable in a control scheme with regards to keeping set-up time low [36] although while a high level of precision is not as important as it would for a FES only system, consistency is still desirable.

The following list prioritises desired factors in a model for FES, indicated by number:

1. Setup time is short.

2. Fatigue is minimised.

3. Control is sufficient for combination with an electromechanical actuator, i.e. the muscle response doesn't have to be perfect but it should not hinder the exoskeleton.

Typically FES systems, commercially and in research, use either amplitude control or pulse-width control. As described in Section 1, there has been some recent investigation into rate-coding as well as modulation of FES recruitment parameters to minimise fatigue [5-8]. The main focus of investigation in this area so far has been focused on controlling pulse-width and frequency together to reduce fatigue. However this work has been focused on isometric and not dynamic contractions [35]. Furthermore, there has been no research investigating control of all three parameters simultaneously (amplitude, pulse-width, frequency). Initial research does indicate the potential for modulation of FES parameters to delay FES-induced muscle fatigue as it can better reflect natural muscle fibre recruitment order [8,37]. For further investigation into parameter modulation of FES to take place, a model is required which enables the muscle response to be predicted to variation of multiple FES parameters simultaneously.

A simple model (Equation 1) has been created based on the relationships described in Section 3 (and approximating the pulse-width relationship as linear) which relates variations in the FES parameters to a change in angle for movement of the elbow joint in the sagittal plane. This model is to be used for input parameters above the threshold where the threshold is defined as the minimum voltage required to cause the elbow to move from rest $\left(0^{\circ}\right.$ - fingers pointing to the floor, palm facing the sagittal plane) to a $20^{\circ}$ bend at a pulse-width and frequency of $200 \mu \mathrm{s}$ and $30.5 \mathrm{~Hz}$ respectively. Thus the threshold voltage causes a $20^{\circ}$ rotation.

$$
\Delta \theta=\mathrm{k}\left(\mathrm{v}_{\mathrm{g}} \Delta \mathrm{v}+\mathrm{pw}_{\mathrm{g}} \Delta \mathrm{pw}+\mathrm{f}_{\mathrm{g}} \Delta \mathrm{f}\right)
$$

Where:

$\theta$ is the elbow angle in degrees

$\mathrm{v}$ is the voltage in volts

$\mathrm{pw}$ is the pulse-width in microseconds (full pulse length $=$ positive + negative portions)

$\mathrm{f}$ is the frequency in Hertz

$\mathrm{k}$ is the overall gain

$\mathrm{v}_{\mathrm{g}}=14$, voltage gain

$\mathrm{pw}_{\mathrm{g}}=0.15$, pulse-width gain

$\mathrm{f}_{\mathrm{g}}=0.22$, frequency gain 
$\mathrm{v}_{\mathrm{g}}, \mathrm{pw}_{\mathrm{g}}$, and $\mathrm{f}_{\mathrm{g}}$ are the individual parameter gains. The values assigned to these gains have been empirically calculated from testing on a single healthy subject. In this work an investigation is conducted into how well these gains, the ratios between them, and the model itself can be used to predict the bicep muscle response to different stimulation input combinations, across different subjects, on different days, and in the presence of fatigue. Further investigation is also conducted into the effect that different FES parameter combinations have on the level of muscle fatigue.

This work seeks to answer the following questions:

1. Can a generalized model be used to predict arm response to FES. For example are all subjects who are more sensitive to voltage steps also more sensitive to pulse-width steps?

2. Can measured individual parameter gains be used to compute responses for the combination steps with a similar accuracy to predicting the responses for the individual steps?

3. How do the responses for each parameter change in the presence of fatigue? For example if the voltage and pulse-width gains decrease within a test does the voltage pulse-width combination proportionally decrease too? Do all the individual parameter gains decrease at the same rate as one another?

4. Can a patient specific model be used across different days? For example if the voltage gain reduces by $50 \%$ between days do the other parameter gains follow a similar trend?

\section{EXPERIMENTAL PROTOCOL}

Ethical approval for all testing was granted by the University of Canterbury Human Ethics Committee. The subjects involved in the tests were young $(20-40$ years old, predominately $<30)$ healthy volunteers. Of the 14 subjects, 6 were female and 8 were male. All were within a healthy weight range. For the purposes of controlling multiple parameters at once, each parameter was controlled so as to contribute the same absolute change in angle. For example, for a $20^{\circ}$ change using both frequency and voltage steps each parameter step should contribute $10^{\circ}$ in change to the overall step.

The testing of the model was performed on 14 different healthy subjects. Subjects were typically tested up to 3 times each day on up to 4 different days. Some subjects were tested less than this depending on availability. On each test day, each subject was assigned a test angle and all three tests for that day were conducted using that angle. The angle which a test subject may be assigned was selected randomly from the following angles: $10^{\circ}$, $15^{\circ}, 25^{\circ}, 40^{\circ}, 60^{\circ}$, or $85^{\circ}$. The selected angle is used to calculate the magnitude of stimulation to be applied in Stage 5 with the aim of that angle being the angle achieved in Stage 5. Each test was at least 10 minutes long to induce muscle fatigue. On a given day, there was no rest time allowed between tests for a particular subject and the next test was started within 2 minutes of the prior test. The set-up procedure and details of the testing is described here in stages.

Stage 1: A large coin sized amount of gel was placed on the electrodes so they were sufficiently coated. This improves comfort and lowers the electrode impedance. Any extra gel which is not on the electrodes is wiped up.

Stage 2: Manual tests are performed in order to check the placement of electrodes. The electrode positions were adjusted until a good comfortable response achieved. This was usually accomplished in less than $20 \mathrm{~s}$.

Stage 3: The exoskeleton is attached. This consists of a metal structure which connects to the arm using Velcro straps and uses a potentiometer at the elbow joint to measure the rotational angle. The voltage threshold tests are then performed once the exoskeleton is securely and comfortably attached. Stimulation is applied at a frequency of $30.5 \mathrm{~Hz}$, and pulse-width of $200 \mu \mathrm{s}$. Voltage steps are applied in increments of $0.5 \mathrm{~V}$ starting at $10 \mathrm{~V}$. Each step is applied for a duration of $3 \mathrm{~s}$ and the peak arm response is recorded in degrees. When a step results in a peak arm angle of $20^{\circ}$ the voltage threshold test is complete and the input voltage is recorded and defined as the threshold voltage. In between each step, if a $20^{\circ}$ angle has not been achieved then the stimulation is turned off for a duration of $3 \mathrm{~s}$ before the next step is applied. This short rest is to prevent the arm getting used to the stimulation which would affect the voltage threshold (more stimulation would be required to achieve a given angle). 
Stage 4: A gain test is performed to determine the value of $\mathrm{k}$ in Equation 1. The level of stimulation is set to the lower stimulation thresholds; $\mathrm{v}=$ Vthresh (voltage found in stage 3), $\mathrm{pw}=200 \mu \mathrm{s}, \mathrm{f}=30.5 \mathrm{~Hz}$.

The voltage is then stepped up in increments of $0.5 \mathrm{~V}$. Each step is applied for a duration of $3 \mathrm{~s}$ and the peak arm response is recorded in degrees. When a step results in a peak arm angle of $80^{\circ}$ the gain test is complete. Equation 1 is used to calculate $\mathrm{k}$. In between each step if an $80^{\circ}$ angle has not been achieved then the stimulation is turned off for a duration of $3 \mathrm{~s}$ before the next step is applied. This short rest is to prevent the arm getting used to the stimulation which would affect the voltage threshold. This completes the set-up procedure.

Stage 5: The main tests are then performed. Initially the threshold stimulation is applied for a duration of $10 \mathrm{~s}$ $\left(\mathrm{v}=\mathrm{v}_{\mathrm{thresh}}, \mathrm{pw}=200 \mu \mathrm{s}, \mathrm{f}=30.5 \mathrm{~Hz}\right)$. A step input is then applied. Step inputs are applied in groups. Each group contains one step input of every parameter combination of the three stimulation parameters ( $v, p w, f, v \& f, p w \&$ $\mathrm{f}, \mathrm{v} \& \mathrm{pw}, \mathrm{v} \& \mathrm{pw} \& \mathrm{f}$ ). $\mathrm{v}, \mathrm{pw}$, and f are referred to as the 'individual steps' while the others are referred to as the 'combination steps'. These steps are always applied in the same order. This should ensure that the change in fatigue between each same step type should be similar for all the step types. Each step is applied for a duration of $10 \mathrm{~s}$. Between each step, the threshold stimulation is applied, also for a duration of $10 \mathrm{~s}$. This test process is repeated until 10 minutes have elapsed at which point the test will complete step tests on the remaining parameter step types in the current group and then stop (for example if 10 minutes have elapsed and the test step type just applied was the pw \& f combination then the test will keep running until it has also applied the $\mathrm{v} \& \mathrm{pw}$ combination and the $\mathrm{v} \&$ pw \& $\mathrm{f}$ combination so each parameter combination will have the same number of step tests).

If any desired step angles require a parameter to exceed its maximum limit, it will instead be applied at its maximum limit. The predicted step sizes used for analysis will be calculated using the measured inputs. An example of the responses observed during the test for one subject test is shown in Figure 13. The angle shown is the peak angle, not the change in angle. It should be noted that Stage 1 and 2 are only performed when then electrodes are first applied. If it is not the first test then Stage 1 and 2 are skipped.

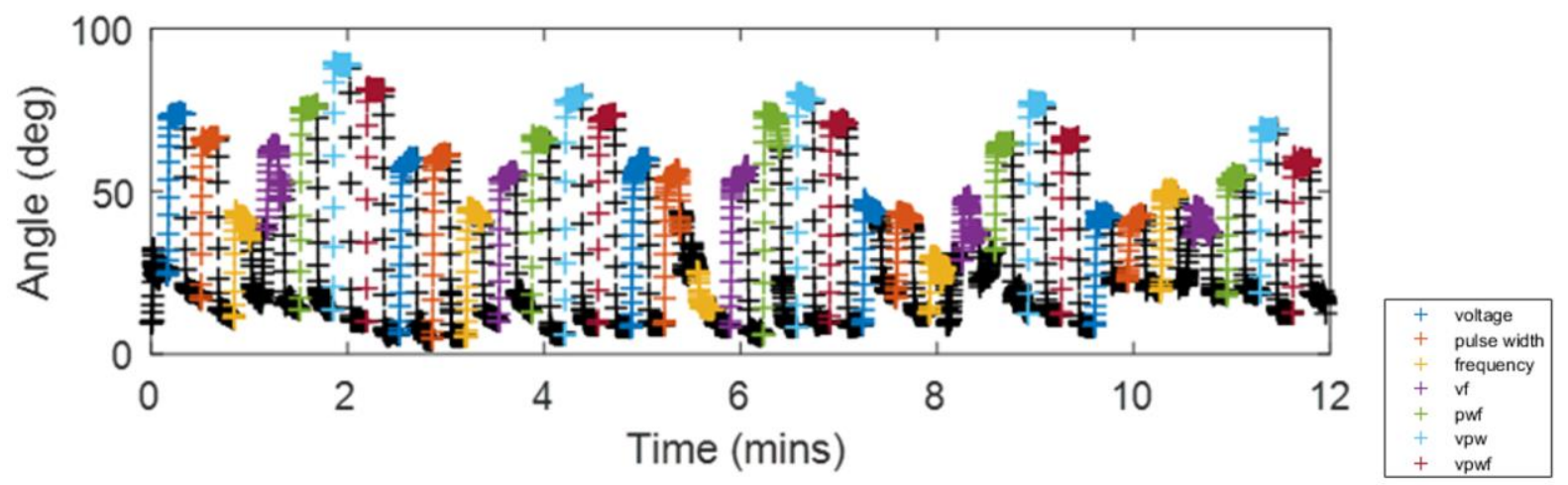

Figure 13: Arm Response to Stimulation Step Tests

14 subjects were tested in total. However, not all completed all tests. Each subject was tested on between $1-4$ days. Each day 3 tests were done on the subject with minimal rest time between. Prior to analysis some tests and subjects were excluded based on the criteria described below.

Some tests were cut short due to discomfort. All tests which lasted less than 10 minutes were excluded. Initially, it was possible for the $\mathrm{k}$ value in Equation 1 to be negative in some cases when the threshold stimulation was very close to the maximum. A limit was placed in the software so that $\mathrm{k}$ could not be less than or equal to zero, and all tests with a negative $\mathrm{k}$ were excluded.

Two subjects had difficulty relaxing their muscles and their arms would sometimes not immediately return to the threshold even after the stimulation was decreased. These two subjects were excluded. Some subjects were not very sensitive to the stimulation and as a result only a very small range of change in angles could be produced from these subjects. These subjects were excluded due to lack of range. 
Some results were excluded due to the arm relaxation angle being too large at the beginning of the threshold test. This was identified by inspection of the arm angle measurements during the threshold test where the initial rest angle was observed to be greater than the threshold angle $\left(20^{\circ}\right)$. Some results which could be placed in this category were included if the responses produced appeared to be consistent with the measured threshold. For example, results with the minimum measured threshold (10 v) which were observed to have no deadband between the measured threshold and increasing intensities of stimulation were included, while those that appeared to have a deadband were excluded. This would only affect methods which use the threshold tests.

After exclusions, data for 8 subjects remained with a total of 50 tests. Subjects were anonymised and assigned codenames (Table 1).

Table 1: List of Subjects and Inclusion/Exclusion Details

\begin{tabular}{|c|c|c|c|c|c|c|}
\hline Subject & $\begin{array}{l}\text { N.o. } \\
\text { Tests }\end{array}$ & $\begin{array}{l}\text { N.o. } \\
\text { Days }\end{array}$ & Excluded & $\begin{array}{l}\text { Post Exclusion } \\
\text { Tests }\end{array}$ & $\begin{array}{l}\text { Post Exclusion } \\
\text { Days }\end{array}$ & Included \\
\hline Alpha & 12 & 4 & 5 & 7 & 4 & yes \\
\hline Bravo & 11 & 4 & 11 & 0 & 0 & \\
\hline Charlie & 9 & 3 & 3 & 6 & 3 & yes \\
\hline Delta & 2 & 1 & 2 & 0 & 0 & \\
\hline Echo & 3 & 1 & 3 & 0 & 0 & \\
\hline Foxtrot & 9 & 3 & 0 & 9 & 3 & yes \\
\hline Golf & 12 & 4 & 11 & 1 & 1 & yes \\
\hline Hotel & 9 & 3 & 9 & 0 & 0 & \\
\hline Indigo & 12 & 4 & 12 & 0 & 0 & \\
\hline Juliett & 12 & 4 & 6 & 6 & 2 & yes \\
\hline Kilo & 12 & 4 & 12 & 0 & 0 & \\
\hline Lima & 6 & 2 & 0 & 6 & 2 & yes \\
\hline Mike & 12 & 4 & 0 & 12 & 4 & yes \\
\hline November & 3 & 1 & 0 & 3 & 1 & yes \\
\hline
\end{tabular}




\section{RESULTS}

To test the ability to predict the combination steps using a linear combination of the gains of the individual parameters, several different methods of prediction were tested. These are described here and a summary of the different methods including results for each is shown in Table 2 .

1. Method 1 used the model (Equation 1) and assumes that the model is the same for different subjects and that each parameter response is similarly affected by fatigue (the individual gains do not vary differently relative to one another over time). The voltage threshold test response was to calculate the overall gain (k) in the model. The model was then used to estimate each step response.

2. Method 2 uses the responses to the first individual parameter steps (voltage, pulse-width, frequency) to calculate the gains for each parameter in the model. The model is then used to estimate the rest of the step responses.

3. Method 3 uses each individual parameter step to calculate the gains for each parameter in the model. These gains are recalculated at each individual parameter step instead of just at the first step. The model is then used to estimate the step response in the next step.

4. Method 4 is similar to Method 3, except that only the voltage steps are used to calculate and adjust the overall model gain (k). Like Method 1 this method assumes that each parameter response was similarly affected by fatigue and that the model is the same for different subjects.

5. Method 5 is similar to Method 2, except that the voltage gain is calculated from the threshold test instead of the first voltage step. The pulse-width and frequency gains are calculated from their respective first steps.

6. Method 6 is similar to Method 2, except that the overall gain $(\mathrm{k})$ is recalculated from the most recent step (step type is ignored). This method assumes that fatigue causes a similar reduction in gains for all parameters.

7. Method 7 is similar to Method 6, except step type is not ignored. i.e. Each vf step is calculated using the individual parameter gains calculated from the first $\mathrm{v}$ and $\mathrm{f}$ steps and the $\mathrm{k}$ value calculated from the previous step of the same type (the last vf step in this case). This method assumes that fatigue causes a different reduction in gain for each parameter.

8. Method 8 is similar to Method 7, except the individual parameter gains are also recalculated based on the previous individual steps ( $\mathrm{v}, \mathrm{pw}, \mathrm{f})$.

9. Method 9 uses the first individual steps to calculate the parameter gains and the first combination steps to calculate a $\mathrm{k}$ value for each combination step type. It then uses the model and these gains selected at the start to calculate all other steps.

10. Method 10 is similar to Method 9, except the threshold test was used to calculate the voltage gain.

11. Method 11 is similar to Method 9, except the individual parameter gains are recalculated based on the previous individual steps ( $\mathrm{v}, \mathrm{pw}, \mathrm{f})$.

12. Method 12 uses Equation 5.1 but recalculates $k$ for each step type based on the previous step of the same type.

13. Method 13 is similar to Method 12, but $\mathrm{k}$ is calculated once for each step type from the first steps.

It should be mentioned that Methods 2, 3,5 - 13 exclude the results from the first group of steps as these methods use the first group to predict future groups. Removing the first group from all results arguably gives a more consistent comparison between methods plus the subject is possibly more relaxed further into the test and thus could be more similar to a non-healthy subject. However doing this had very little effect on the results and it does not change the overall conclusions so for the methods not using the first group for predictions, the results for the first steps have been included.

A comparison of the predicted change in angle compared with the measured change in angle for all data points for each method is shown in Figure 14 to Figure 21. 


\begin{tabular}{|c|c|c|c|c|c|}
\hline Method & Gains & $\mathrm{k}$ & $\mathrm{R}^{2}$ & Gradient & $\begin{array}{l}\text { Auto- } \\
\text { regressive? }\end{array}$ \\
\hline 1 & $14,0.15,0.22$ & Calculated from $v_{\text {thresh }}$ test & 0.3659 & 0.6 & no \\
\hline 2 & $\begin{array}{l}\text { Calculated from first } v \text {, } \\
\text { pw, f steps }\end{array}$ & 1 & 0.54526 & 0.77 & no \\
\hline 3 & $\begin{array}{l}\text { Calculated from previous } \\
\mathrm{v}, \mathrm{pw}, \mathrm{f} \text { steps }\end{array}$ & 1 & 0.60422 & 0.69 & gains \\
\hline 4 & $14,0.15,0.22$ & Calculated from previous $\mathrm{v}$ step & 0.39111 & 0.6 & $\mathrm{k}$ \\
\hline 5 & $\begin{array}{l}\text { Calculated from vthresh } \\
\text { test and first pw, f steps }\end{array}$ & 1 & 0.52253 & 0.76 & no \\
\hline 6 & $\begin{array}{l}\text { Calculated from first } v \text {, } \\
\text { pw, f steps }\end{array}$ & $\begin{array}{l}\text { Calculated from the previous } \\
\text { step (ignores step type) }\end{array}$ & 0.2265 & 0.6 & $\mathrm{k}$ \\
\hline 7 & $\begin{array}{l}\text { Calculated from first } v \text {, } \\
\text { pw, f steps }\end{array}$ & $\begin{array}{l}\text { Calculated from the previous } \\
\text { same step type }\end{array}$ & 0.75192 & 0.93 & $\mathrm{k}$ \\
\hline 8 & $\begin{array}{l}\text { Calculated from previous } \\
\mathrm{v}, \mathrm{pw}, \mathrm{f} \text { steps }\end{array}$ & $\begin{array}{l}\text { Calculated from the previous } \\
\text { same step type }\end{array}$ & 0.75299 & 0.93 & gains and $\mathrm{k}$ \\
\hline 9 & $\begin{array}{l}\text { Calculated from first } v \text {, } \\
\text { pw, f steps }\end{array}$ & $\begin{array}{l}1 \text { for } \mathrm{v}, \mathrm{pw}, \mathrm{f} \text { steps. Calculated } \\
\text { from first step of same type for } \\
\text { combinations }\end{array}$ & 0.6546 & 0.95 & no \\
\hline 10 & $\begin{array}{l}\text { Calculated from } \\
\mathrm{v}_{\text {thresh }} \text { test and first pw, f } \\
\text { steps }\end{array}$ & $\begin{array}{l}1 \text { for } \mathrm{v}, \mathrm{pw}, \mathrm{f} \text { steps. Calculated } \\
\text { from first step of same type for } \\
\text { combinations }\end{array}$ & 0.62329 & 0.93 & no \\
\hline 11 & $\begin{array}{l}\text { Calculated from previous } \\
\mathrm{v}, \mathrm{pw}, \mathrm{f} \text { steps }\end{array}$ & $\begin{array}{l}1 \text { for } \mathrm{v}, \mathrm{pw}, \mathrm{f} \text { steps. Calculated } \\
\text { from first step of same type for } \\
\text { combinations }\end{array}$ & 0.54037 & 0.86 & gains \\
\hline 12 & $14,0.15,0.22$ & $\begin{array}{l}\text { Calculated from the previous } \\
\text { same step type }\end{array}$ & 0.76105 & 0.93 & $\mathrm{k}$ \\
\hline 13 & $14,0.15,0.22$ & $\begin{array}{l}\text { Calculated from the first same } \\
\text { step type }\end{array}$ & 0.66045 & 0.93 & no \\
\hline
\end{tabular}


(a)

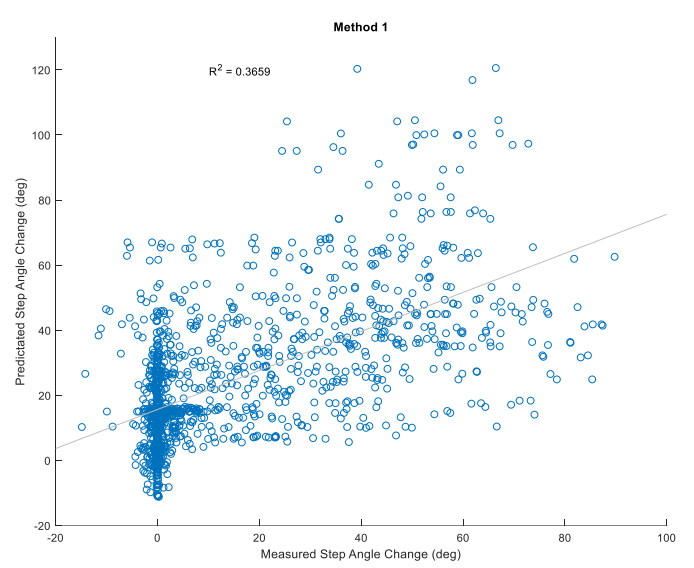

(b)

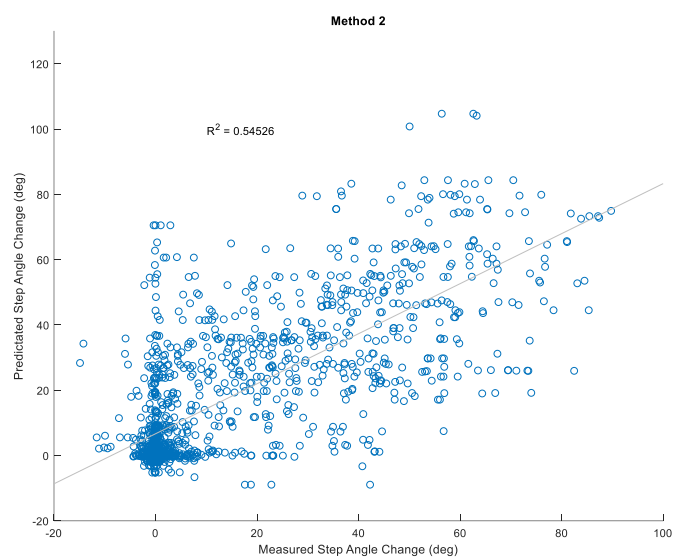

Figure 14: Method 1 (a) and Method 2 (b), Measured Change in Angle Compared to Predicted

(a)

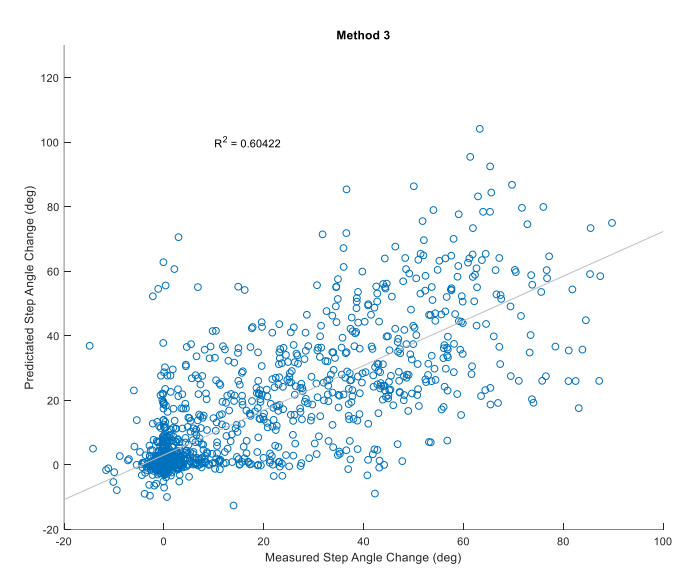

Figure 15: Method 3(a) and Method 4(b), Measured Change in Angle Compared to Predicted (b)

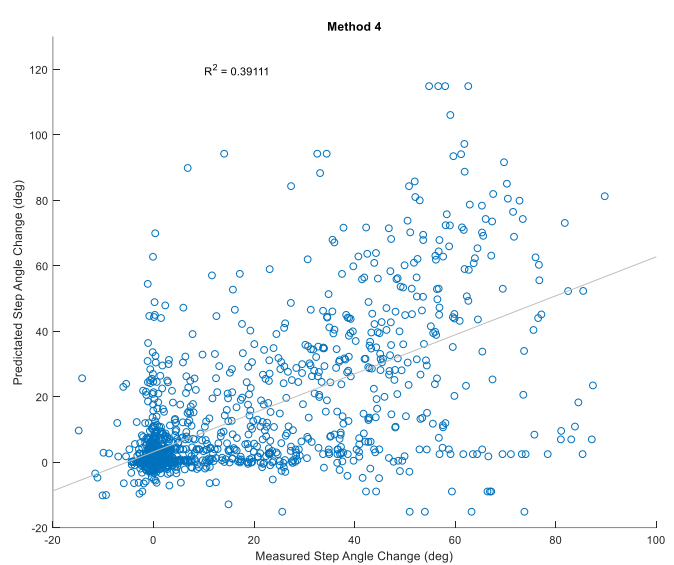

(a)

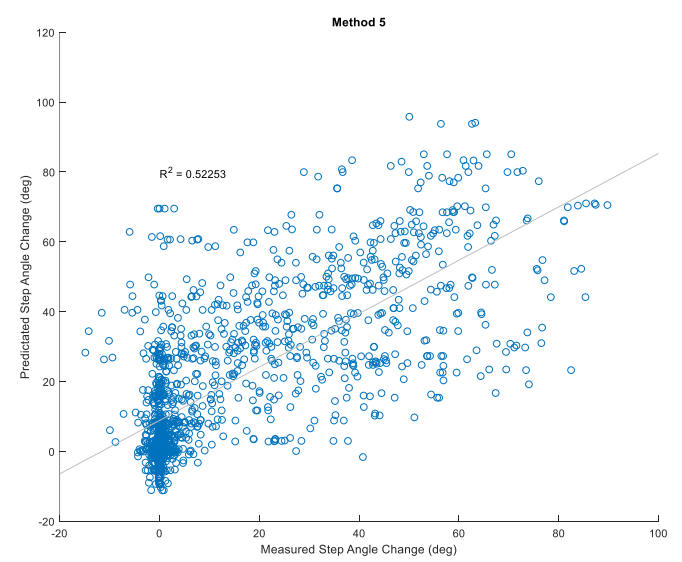

(b)

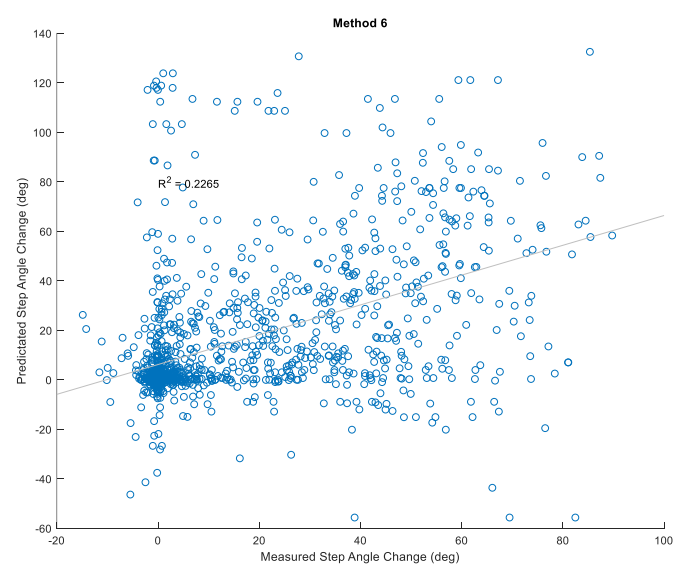

Figure 16: Method 5 (a) and Method $6(b)$, Measured Change in Angle Compared to Predicted 
(a)

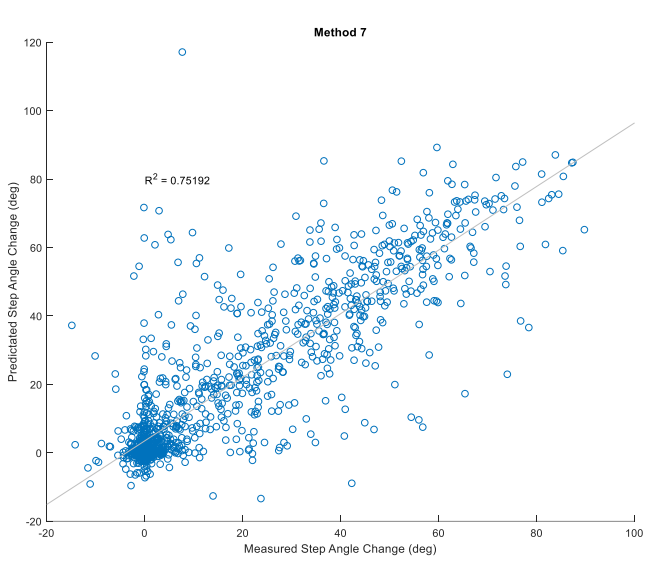

(b)

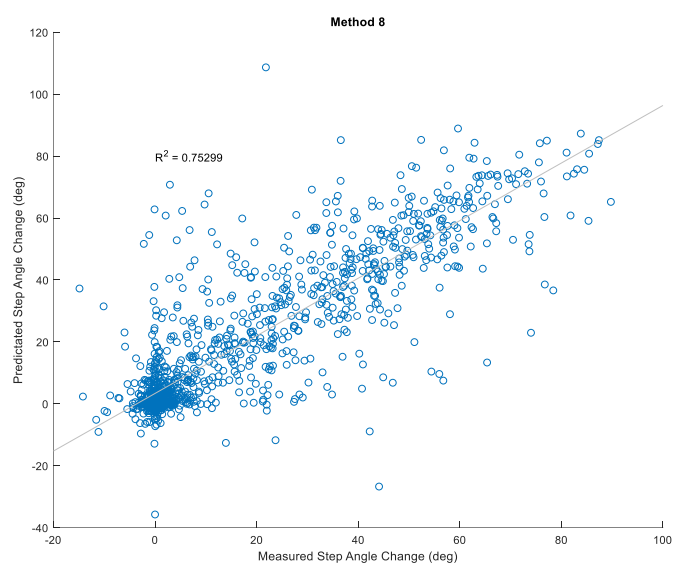

Figure 17: Method $7(a)$ and Method $8(b)$, Measured Change in Angle Compared to Predicted

(a)

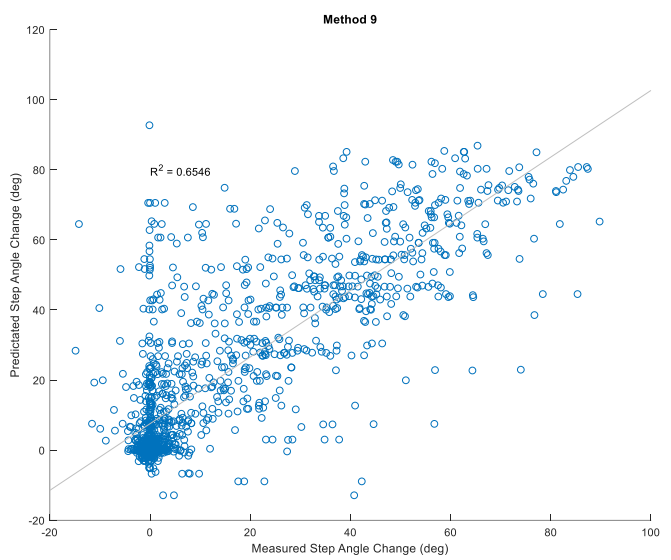

(b)

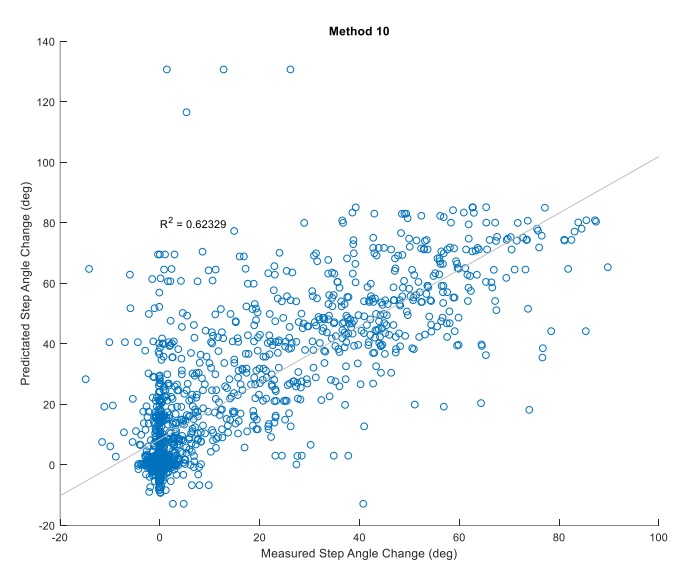

Figure 18: Method 9 (a) and Method $10($ b), Measured Change in Angle Compared to Predicted

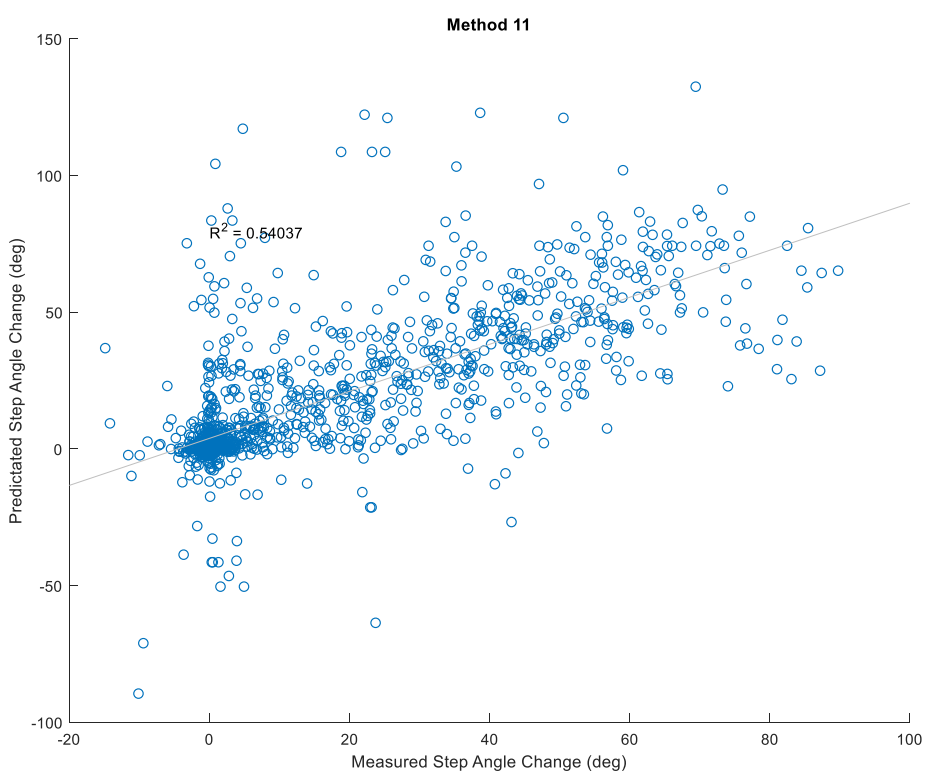

Figure 19: Method 11, Measured Change in Angle Compared to Predicted 


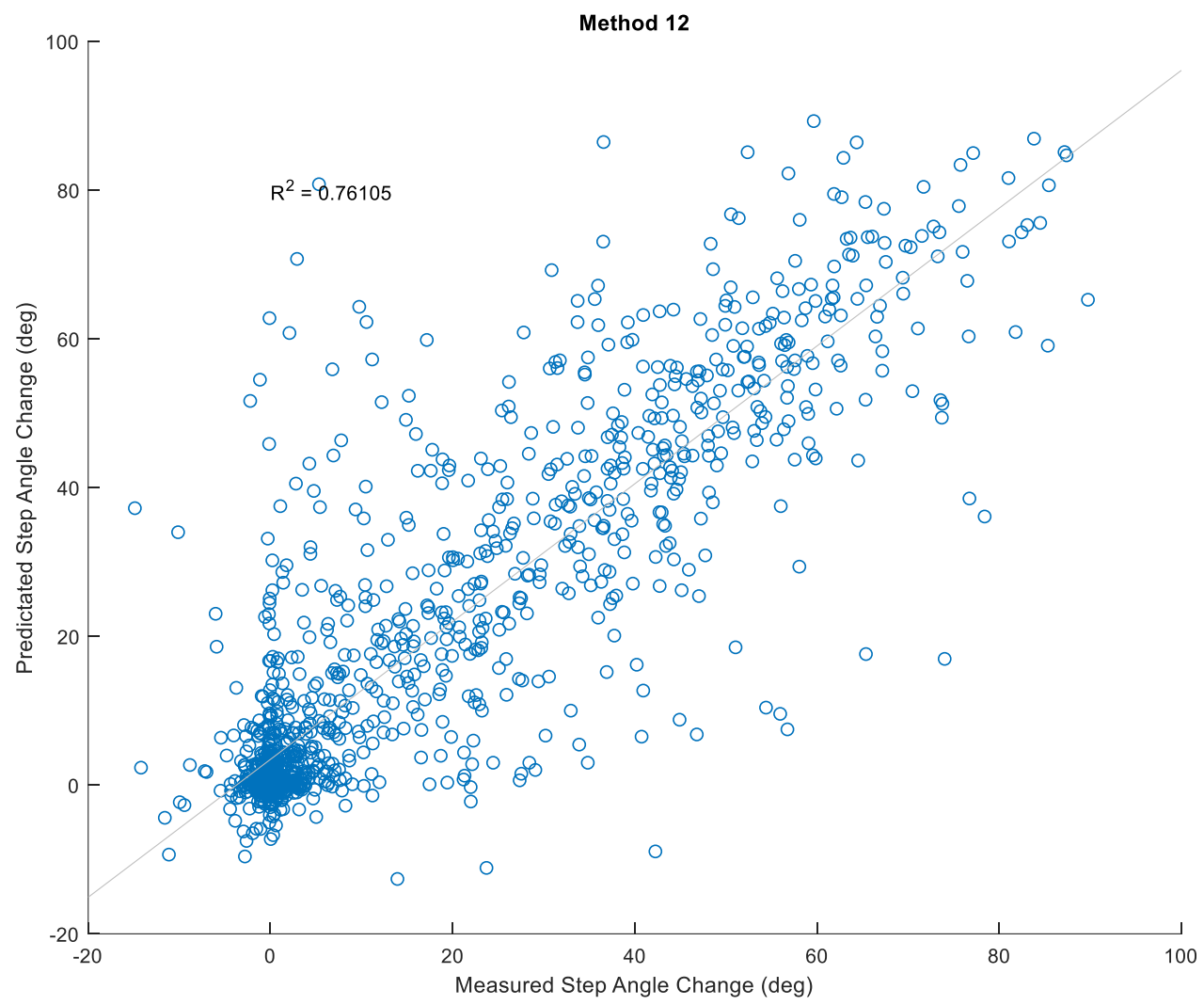

Figure 20: Method 12, Measured Change in Angle Compared to Predicted

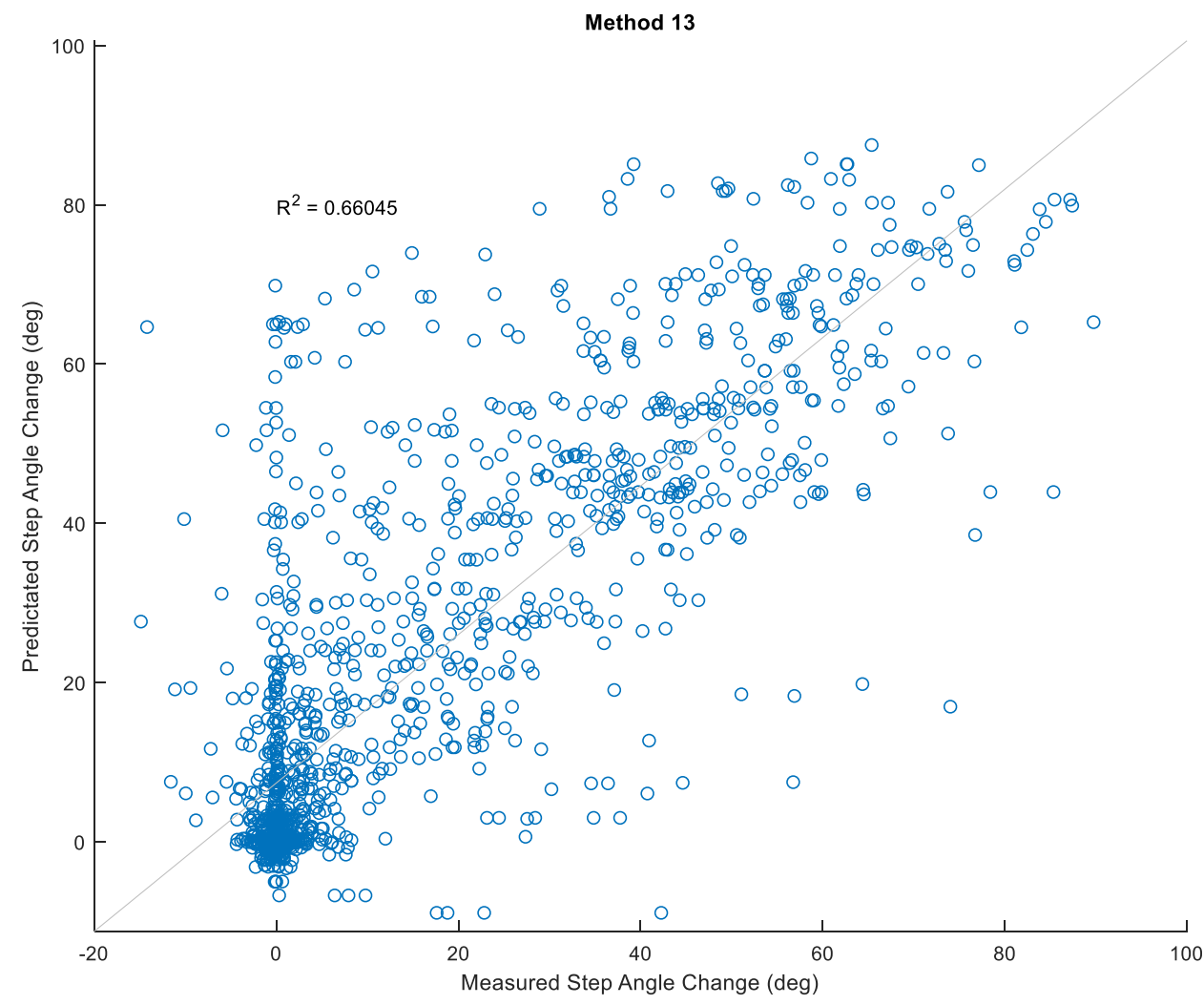

Figure 21: Method 13, Measured Change in Angle Compared to Predicted 
A comparison of $\mathrm{R}^{\mathbf{2}}$ and gradient values for each method by subject and by parameter is shown in Table 3 to Table 6 . $\mathrm{R}^{2}$ values greater than 0.6 , and gradients between 0.9 and 1.1 , are highlighted in green.

Table 3: $R^{2}$ for Each Method by Parameter

\begin{tabular}{|c|c|c|c|c|c|c|c|c|c|c|c|c|c|}
\hline $\mathrm{R}^{2}$ & Method 1 & Method 2 & Method 3 & Method 4 & Method 5 & Method 6 & Method 7 & Method 8 & Method 9 & Method 10 & Method 11 & Method 12 & Method 13 \\
\hline $\mathrm{v}$ & 0.3701 & 0.3720 & 0.6496 & 0.6496 & 0.3751 & 0.2384 & 0.6496 & 0.6496 & 0.3720 & 0.3751 & 0.6496 & 0.6496 & 0.3720 \\
\hline $\mathrm{pw}$ & 0.2933 & 0.6320 & 0.6977 & 0.1566 & 0.6257 & 0.1050 & 0.6273 & 0.6987 & 0.6320 & 0.6257 & 0.6977 & 0.6877 & 0.6283 \\
\hline $\mathrm{f}$ & 0.0641 & 0.2788 & 0.1477 & 0.1341 & 0.2692 & 0.0900 & 0.1537 & 0.1547 & 0.2788 & 0.2692 & 0.1477 & 0.1552 & 0.2767 \\
\hline vf & 0.2444 & 0.4840 & 0.5684 & 0.4477 & 0.4010 & 0.0756 & 0.7762 & 0.7538 & 0.7639 & 0.7755 & 0.4881 & 0.7555 & 0.7691 \\
\hline pwf & 0.3471 & 0.7210 & 0.7116 & 0.2754 & 0.7250 & 0.3021 & 0.7760 & 0.7885 & 0.6467 & 0.6575 & 0.5190 & 0.7774 & 0.6968 \\
\hline vpw & 0.3755 & 0.5230 & 0.5511 & 0.4712 & 0.4859 & 0.2173 & 0.7689 & 0.7631 & 0.6545 & 0.6485 & 0.3924 & 0.7600 & 0.6430 \\
\hline vpwf & 0.2677 & 0.5331 & 0.5460 & 0.4246 & 0.4855 & 0.5842 & 0.8105 & 0.7523 & 0.7039 & 0.5520 & 0.5029 & 0.8266 & 0.6904 \\
\hline
\end{tabular}

Table 4: Gradient of the Fitted Line for Each Method by Parameter

\begin{tabular}{|c|c|c|c|c|c|c|c|c|c|c|c|c|c|}
\hline Gradient & Method 1 & Method 2 & Method 3 & Method 4 & Method 5 & Method 6 & Method 7 & Method 8 & Method 9 & Method 10 & Method 11 & Method 12 & Method 13 \\
\hline $\mathrm{v}$ & 0.69 & 0.65 & 0.85 & 0.85 & 0.69 & 0.86 & 0.85 & 0.85 & 0.65 & 0.69 & 0.85 & 0.85 & 0.65 \\
\hline pw & 0.59 & 0.99 & 0.9 & 0.43 & 0.98 & 0.46 & 0.9 & 0.91 & 0.99 & 0.98 & 0.9 & 0.91 & 0.98 \\
\hline $\mathrm{f}$ & 0.31 & 1.1 & 0.53 & 0.28 & 1.1 & 0.84 & 0.54 & 0.55 & 1.1 & 1.1 & 0.53 & 0.55 & 1 \\
\hline vf & 0.5 & 0.76 & 0.71 & 0.61 & 0.76 & 0.38 & 0.95 & 0.95 & 1.1 & 1 & 0.9 & 0.94 & 1 \\
\hline pwf & 0.52 & 1 & 0.8 & 0.5 & 1 & 0.65 & 0.96 & 0.96 & 0.99 & 0.99 & 0.82 & 0.96 & 0.97 \\
\hline vpw & 0.58 & 0.6 & 0.57 & 0.69 & 0.59 & 0.43 & 0.89 & 0.89 & 0.85 & 0.84 & 0.79 & 0.87 & 0.83 \\
\hline vpwf & 0.46 & 0.7 & 0.59 & 0.63 & 0.69 & 0.99 & 0.95 & 0.95 & 0.95 & 0.92 & 0.85 & 0.95 & 0.92 \\
\hline
\end{tabular}

Table 5: $R^{2}$ for Each Method by Subject

\begin{tabular}{|c|c|c|c|c|c|c|c|c|c|c|c|c|c|}
\hline $\mathrm{R}^{2}$ & Method 1 & Method 2 & Method 3 & Method 4 & Method 5 & Method 6 & Method 7 & Method 8 & Method 9 & Method 10 & Method 11 & Method 12 & Method 13 \\
\hline All & 0.3659 & 0.5453 & 0.6042 & 0.3911 & 0.5225 & 0.2265 & 0.7519 & 0.7530 & 0.6546 & 0.6233 & 0.5404 & 0.7611 & 0.6604 \\
\hline Alpha & 0.3907 & 0.3562 & 0.4584 & 0.0495 & 0.4326 & 0.2504 & 0.7438 & 0.7379 & 0.5129 & 0.5498 & 0.4191 & 0.7355 & 0.5124 \\
\hline Charlie & 0.4141 & 0.2804 & 0.3307 & 0.4155 & 0.2171 & 0.0858 & 0.7810 & 0.7092 & 0.7159 & 0.6624 & 0.4639 & 0.7794 & 0.7188 \\
\hline Foxtrot & 0.3591 & 0.4032 & 0.4689 & 0.2697 & 0.5376 & 0.1779 & 0.5792 & 0.5788 & 0.4629 & 0.5100 & 0.4224 & 0.5760 & 0.4654 \\
\hline Golf & 0.7140 & 0.7190 & 0.6716 & 0.0027 & 0.7226 & 0.0037 & 0.8565 & 0.8565 & 0.7041 & 0.7138 & 0.6783 & 0.8719 & 0.7580 \\
\hline Juliett & 0.6992 & 0.6926 & 0.7209 & 0.6466 & 0.6156 & 0.2291 & 0.8685 & 0.8686 & 0.7921 & 0.7483 & 0.6369 & 0.8689 & 0.7954 \\
\hline Lima & 0.3280 & 0.1499 & 0.1660 & 0.2464 & 0.2771 & 0.0136 & 0.4879 & 0.4950 & 0.4268 & 0.1773 & 0.0856 & 0.3209 & 0.4189 \\
\hline Mike & 0.1488 & 0.1435 & 0.2774 & 0.4093 & 0.1281 & 0.0346 & 0.3412 & 0.4142 & 0.2540 & 0.2673 & 0.2194 & 0.4275 & 0.2914 \\
\hline November & 0.1298 & 0.5372 & 0.5149 & 0.2609 & 0.3494 & 0.0789 & 0.4701 & 0.4646 & 0.5423 & 0.3103 & 0.4985 & 0.4691 & 0.5277 \\
\hline
\end{tabular}

Table 6: Gradient of the Fitted Line for Each Method by Subject

\begin{tabular}{|c|c|c|c|c|c|c|c|c|c|c|c|c|c|}
\hline Gradient & Method 1 & Method 2 & Method 3 & Method 4 & Method 5 & Method 6 & Method 7 & Method 8 & Method 9 & Method 10 & Method 11 & Method 12 & Method 13 \\
\hline All & 0.6 & 0.77 & 0.69 & 0.6 & 0.76 & 0.6 & 0.93 & 0.93 & 0.95 & 0.93 & 0.86 & 0.93 & 0.93 \\
\hline Alpha & 0.24 & 0.5 & 0.51 & 0.15 & 0.51 & 0.52 & 0.86 & 0.86 & 0.67 & 0.67 & 0.67 & 0.85 & 0.64 \\
\hline Charlie & 0.6 & 0.46 & 0.36 & 0.52 & 0.43 & 0.46 & 0.98 & 0.94 & 1.2 & 1.1 & 0.89 & 0.97 & 1.1 \\
\hline Foxtrot & 0.82 & 0.71 & 0.76 & 0.74 & 0.72 & 0.62 & 0.8 & 0.8 & 0.68 & 0.65 & 0.75 & 0.8 & 0.68 \\
\hline Golf & 0.19 & 0.86 & 0.71 & 0.003 & 0.86 & 0.051 & 1 & 1 & 1.1 & 1.1 & 0.86 & 1.1 & 0.98 \\
\hline Juliett & 0.37 & 0.64 & 0.64 & 0.77 & 0.44 & 0.55 & 0.99 & 0.99 & 1 & 0.96 & 1 & 0.99 & 1 \\
\hline Lima & 2.1 & 0.38 & 0.25 & 0.41 & 1.4 & 0.2 & 0.75 & 0.75 & 0.86 & 0.85 & 0.68 & 0.75 & 0.86 \\
\hline Mike & 0.54 & 0.42 & 0.45 & 0.76 & 0.38 & 0.49 & 0.82 & 0.87 & 0.77 & 0.76 & 0.86 & 0.81 & 0.78 \\
\hline November & 0.4 & 0.78 & 0.66 & 0.36 & 0.71 & 0.3 & 0.77 & 0.76 & 0.92 & 0.84 & 0.77 & 0.77 & 0.9 \\
\hline
\end{tabular}




\section{DISCUSSION}

The results from testing of the FES model described by Equation 1, in Section 4, are discussed here in relation to the questions stated previously also in Section 4.

1. Can a generalized model be used to predict arm response to FES. For example are all subjects who are more sensitive to voltage steps also more sensitive to pulse-width steps?

The answer to this question can be found by comparing the methods which compute subject specific parameter gains with the similar methods which use parameter gains defined in Equation 1. Comparing $\mathrm{R}^{2}$ for Method 7 with Method 12, and $\mathrm{R}^{2}$ for Method 9 with Method 13, indicates that there is very little difference in the predictive ability of the model between using measured individual gains for each subject and using the predefined individual parameter gains. Indeed, the predefined gains give a slightly better performance although the difference is not observed to be large. What this suggests is that if Subject A is more sensitive to pulse-with increases compared with Subject B then Subject A will also be more sensitive to voltage increases compared to the Subject B.

It is promising to note that the method which performs the best for most subjects (and in general) does not require measurement of the individual parameter gains. Instead only the overall gain $(\mathrm{k})$ needs to be measured. This is important because the less measurements required during setup means a shorter setup time which greatly improves the ability of the model to be clinically useful. It is important to note however that the model will still need some subject specific measurements. This is because how the overall gain $(\mathrm{k})$ is calculated greatly affects the predictive ability of the model and $\mathrm{k}$ is not independent from subject or parameter step type. This shall be further discussed in relation to the next two questions.

2. Can measured individual parameter gains be used to compute responses for the combination steps with a similar accuracy to predicting the responses for the individual steps?

Methods, 2, 3, and 5 all calculate the individual parameter gains using different methods and use a linear combination of these to predict the outputs for the combination steps. In all cases, $\mathrm{k}$ is assumed to be 1 . All three of these methods produce predictions with an $\mathrm{R}^{2}$ greater than 0.5 . Furthermore, each combination step produces a better prediction than the individual voltage and frequency steps (Table 3). Thus a linear model is no worse at predicting the response to a combination step than it is to predicting a response to an individual parameter step.

However, assigning an overall gain $(\mathrm{k})$ to each individual step produced much better predictions as can be seen by comparing the $\mathrm{R}^{2}$ for Method 2 with the $\mathrm{R}^{2}$ for Method 9 , and the $\mathrm{R}^{2}$ for Method 5 with the $\mathrm{R}^{2}$ for Method 11 which suggests that the response to an individual parameter step plus the response to a different individual parameter step is not quite the same as the response to a combination of those. In general, combination steps have better prediction results compared to the individual steps. This is especially true for voltage and frequency, the latter of which is the most difficult to predict. This may be as a result of the difficulty in producing large responses using these parameters on their own.

Pulse-width, despite its linear approximation (as described in Section 3) produces good predictions for individual steps and for cases where the overall gain (k) is not considered pulse-width step predictability only sees an improvement when combined with frequency. When the overall gain $(\mathrm{k})$ is considered all of the combination steps result in better predictions using the model than any of the individual steps. It should be noted that the pulse-width frequency combination step is the only combination step that is not improved by accounting for the overall gain (k). Thus, the output from a pulse-width frequency combination step could be considered as a linear combination of the outputs from each individual pulse-width and frequency step. If however, voltage is to be used as part of a combination step, then the overall gain (k) must be taken into account. 
From a practical perspective, the results related to this question are not very useful. This is because it has already been demonstrated in the discussion related to question 1 that simply using the overall gain $(\mathrm{k})$ and predefined individual gains produces very good predictions. Indeed, comparing $\mathrm{R}^{2}$ for Method 11 , which uses information from previous steps to recalculate the gains, with Methods 7 and 12 which do the same but with $\mathrm{k}$ instead, and Method 8 which uses both, clearly demonstrates that predictability is not improved by calculating both the individual gains and $\mathrm{k}$ compared with only calculating $\mathrm{k}$. Furthermore using information from previous steps to recalculate the individual gains and not $\mathrm{k}$ can produce worse results than having no auto-regressive behaviour at all, as seen by comparing $\mathrm{R}^{2}$ for Method 10 with Methods 3 and 11 . The reason for this is best discussed in relation to the next question.

3. How do the responses for each parameter change in the presence of fatigue? For example if the voltage and pulse-width gains decrease within a test does the voltage pulse-width combination proportionally decrease too? Do all the individual parameter gains decrease at the same rate as one another?

As may have been noticed, there is an exception to the above described rule that calculating the overall gain $(\mathrm{k})$ produces better results than when $\mathrm{k}$ is not considered. The exception to this is seen in Method 11. In Method $11 \mathrm{k}$ is calculated once for each step type using the first steps for each step type. However, auto-regressive behaviour is performed for the individual parameter gains. If the parameter step types were not affected differently by fatigue then it would be expected that Method 11 would produce no worse a prediction than Method 3 however this is not the case suggesting that the responses for the different step types are indeed affected differently by fatigue, or comparatively, that the different step types induce different amounts of fatigue. This implies that the individual gains should not be used to predict the combination step outputs in the presence of fatigue. Even the pulse-width frequency combination step is no exception here.

This is further backed up by the poor predictions produced when step type is not taken into account when calculating $\mathrm{k}$, as is the case for Methods 1, 4, and 6, which all produce poor predictions compared to the other methods. It is very important that when $\mathrm{k}$ is calculated and used it is always for the same step type.

To compare how much each step type is affected by muscle fatigue, $\mathrm{R}^{2}$ for Method 7 can be compared with Method 9, and $\mathrm{R}^{2}$ for Method 12 can be compared with Method 13. Essentially what this does is compare methods which perform auto-regressive behaviour with methods that don't. It is expected that of these methods, those using auto-regressive behaviour will produce better predictions (Methods 7 and 12). It is how much better these methods are compared to those without auto-regressive behaviour which will give insight as to which step types are more consistent in the presence of fatigue. Any step type which shows a large difference is likely more affected by (and thus likely induces more) muscle fatigue. The way that muscle fatigue has been defined in this paper is with regards to the ability of the muscle to perform a contraction for a given electrode position. Muscle fatigue is defined this way because this is a clinically relevant definition and an easy one to measure. Alternatively muscle fatigue could be viewed from a muscle fibre level. During this test it is probable that some step types induce fatigue in muscle fibres recruited during other step types and thus affect the response of other step types. In this case the fatigue of the shared muscle fibres should similarly affect the response for each step type so any step type with a larger variation in response possibly does induce more fatigue but this is not certain, and the order of the steps may affect how the different steps impact one another's response despite the short rest times between. Regardless, from a clinical perspective, any step type which performs more consistently for a given electrode position and is less affected by other step types is more useful for control of FES. Thus, step types which show the least amount of change in predictability for these comparisons are more desirable. The differences in predictability for each step type for the described methods are shown in Table 7. Predictions with an absolute $\mathrm{R}^{2}$ change of greater than 0.1 are highlighted in red. Predictions with an average $\mathrm{R}^{\mathbf{2}}$ difference of magnitude less than 0.1 are highlighted in green, those with an average difference of between a magnitude of 0.1 and 0.2 are highlighted in yellow, and those with an average difference of greater than magnitude 0.2 are highlighted in red. Likewise, predictions with a gradient difference greater than a magnitude of 0.1 are highlighted in red. Predictions with an average gradient difference of magnitude less than 0.1 are highlighted in green, and those with an average difference of magnitude greater than 0.1 are highlighted in red. 
Table 7: Comparison between Methods with Auto-regressive Behaviour and Without for Different Step Types

\begin{tabular}{|c|c|c|c|}
\hline \multirow[b]{2}{*}{$\mathrm{R}^{2}$} & $\begin{array}{l}\text { No Auto-regressive } \\
\text { Behaviour }\end{array}$ & $\begin{array}{l}\text { Auto- } \\
\text { regressive } \\
\text { Behaviour }\end{array}$ & \\
\hline & Method 9 & Method 7 & Difference \\
\hline $\mathrm{v}$ & 0.3720 & 0.6496 & 0.2776 \\
\hline pw & 0.6320 & 0.6273 & -0.0047 \\
\hline$f$ & 0.2788 & 0.1537 & -0.1251 \\
\hline vf & 0.7639 & 0.7762 & 0.0123 \\
\hline pwf & 0.6467 & 0.7760 & 0.1293 \\
\hline vpw & 0.6545 & 0.7689 & 0.1144 \\
\hline vpwf & 0.7039 & 0.8105 & 0.1065 \\
\hline
\end{tabular}

\begin{tabular}{|c|c|c|}
\hline $\begin{array}{l}\text { No Auto-regressive } \\
\text { Behaviour }\end{array}$ & $\begin{array}{l}\text { Auto- } \\
\text { regressive } \\
\text { Behaviour }\end{array}$ & \\
\hline Method 13 & Method 12 & Difference \\
\hline 0.3720 & 0.6496 & 0.2776 \\
\hline 0.6283 & 0.6877 & 0.0594 \\
\hline 0.2767 & 0.1552 & -0.1215 \\
\hline 0.7691 & 0.7555 & -0.0136 \\
\hline 0.6968 & 0.7774 & 0.0806 \\
\hline 0.6430 & 0.7600 & 0.1170 \\
\hline 0.6904 & 0.8266 & 0.1362 \\
\hline
\end{tabular}

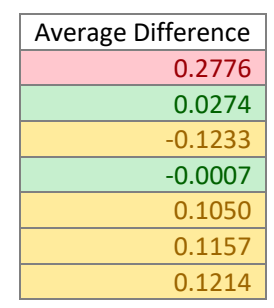

\begin{tabular}{|l|rr|r|r|}
\cline { 2 - 4 } \multicolumn{1}{c|}{ Gradient } & Method 9 & Method 7 & Difference \\
\hline v & 0.65 & 0.85 & 0.20 \\
\hline pw & 0.99 & 0.90 & -0.09 \\
\hline f & 1.10 & 0.54 & -0.56 \\
\hline vf & 1.10 & 0.95 & -0.15 \\
\hline pwf & 0.99 & 0.96 & -0.03 \\
\hline vpw & 0.85 & 0.89 & 0.04 \\
\hline vpwf & 0.95 & 0.95 & 0.00 \\
\hline
\end{tabular}

\begin{tabular}{|c|c|c|}
\hline Method 13 & Method 12 & Difference \\
\hline 0.65 & 0.85 & 0.20 \\
\hline 0.98 & 0.91 & -0.07 \\
\hline 1.00 & 0.55 & -0.45 \\
\hline 1.00 & 0.94 & -0.06 \\
\hline 0.97 & 0.96 & -0.01 \\
\hline 0.83 & 0.87 & 0.04 \\
\hline 0.92 & 0.95 & 0.03 \\
\hline
\end{tabular}

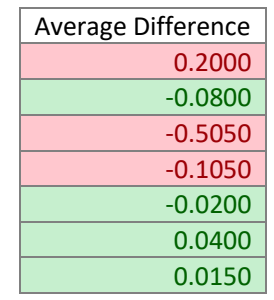

It is important to also consider the overall precision and accuracy of the predictions as well as the change in precision and accuracy. Frequency steps are very difficult to predict precisely and have a lot of variation in their responses even within a specific subject. The poor predictability of the frequency steps may be because it is difficult to produce large movements using frequency on its own. In general, the ranges covered were attempted to be kept similar but this is difficult to do, especially for frequency. Thus there is some variation in the ranges which will likely affect the results. Another issue is that it was assumed that a larger frequency would produce a larger response [21] however saturation occurred lower than was expected. Sometimes, an increased frequency was observed to result in a decreased arm response. Regardless, all of these difficulties contribute to a strong argument for not using increases in frequency on its own for FES movements.

In general, combining frequency or any combination of parameters did result in better predictions. However, in the presence of fatigue the results from the combination steps are generally not any better than using pulse-width on its own. On the other hand, the ability to predict the voltage steps without auto-regressive behaviour was greatly reduced in the presence of fatigue. This suggests that it is the voltage gain in particular that varies most during fatigue and affects the predictions of the combination steps if only the recalculated individual gains are used without $\mathrm{k}$. This also explains why the pulse-width frequency combination step outperformed the other combination steps in the same conditions. When voltage is combined with the other parameters the gains do not decrease as quickly in comparison with using voltage on its own.

It is important to note that no comment can be made with regards to using current steps as for this study a voltage controlled FES device was used. It is established that current controlled FES is more predictable than voltage controlled FES, so it is possible that current steps also perform better in the presence of fatigue then voltage. As to how the combination steps would compare for a current controlled stimulator this is unknown. As of yet, no investigation has been done into the effect of current vs voltage controlled stimulators on fatigue which the authors are aware of.

Overall the combination steps perform better than the voltage and frequency steps and similarly to the pulse-width steps when muscle fatigue is considered.

4. Can a patient specific model be used across different days? For example if the voltage gain reduces by $50 \%$ between days do the other parameter gains follow a similar trend?

Already it has been shown that the same gains work for different subjects and thus so too must they work for different days. However $\mathrm{k}$ has been shown to be specific to a step type, individual, and fatigue so it can be concluded that $\mathrm{k}$ will vary across days as well. 


\section{CONCLUSION}

Overall there does seem to be an argument for using combination steps over the individual steps with regards to control improvements for voltage controlled devices. Using pulse-width on its own did produced comparatively good results especially when muscle fatigue is considered where it outperformed the combination steps, while voltage and frequency individual steps were generally not great on their own from either a control or fatigue perspective. Combining parameters does have the advantage of increasing the range of movement which may be achieved while still producing a consistent response in the presence of muscle fatigue.

Good predictions of angle response to input parameters were achieved for a range of subjects across different days and tests using Equation 1. Precise control can be achieved by setting the individual gains to an approximate magnitude and adjusting $\mathrm{k}$ in response to changes. Each step type should have its own overall gain (k). Only the overall gain needs to be measured for the model as the individual parameter gains generalise across subjects which means setup time can be kept short as is highly desirable for stroke rehabilitation tools.

Funding: This work was supported by the University of Canterbury

\section{REFERENCES}

1. Peckham, P.H. and J.S. Knutson, Functional Electrical Stimulation for Neuromuscular Applications. Annual Review of Biomedical Engineering, 2005. 7: p. 327-360.

2. Sweeney, H.L., Gene Doping, in Scientific American, . July 2004, Scientific American, a division of Nature America, Inc.

3. Maffiuletti, N.A., Physiological and methodological considerations for the use of neuromuscular electrical stimulation. European journal of applied physiology, 2010. 110(2): p. 223-234.

4. Henneman, E., Relation between size of neurons and their susceptibility to discharge. Science, 1957. 126(3287): p. 1345-1347.

5. Chou, L.-W., et al., The effectiveness of progressively increasing stimulation frequency and intensity to maintain paralyzed muscle force during repetitive activation in persons with spinal cord injury. Archives of physical medicine and rehabilitation, 2008. 89(5): p. 856-864.

6. Doll, B.D., et al., Dynamic optimization of stimulation frequency to reduce isometric muscle fatigue using a modified Hill-Huxley model. Muscle \& nerve, 2018. 57(4): p. 634-641.

7. Rouhani, H., et al., Identification of ankle plantar-flexors dynamics in response to electrical stimulation. Medical engineering \& physics, 2016. 38(11): p. 1166-1171.

8. $\quad$ Barss, T.S., et al., Utilizing Physiological Principles of Motor Unit Recruitment to Reduce Fatigability of Electrically-Evoked Contractions: A Narrative Review. Archives of physical medicine and rehabilitation, 2018. 99(4): p. 779-791.

9. $\quad$ Stewart, A.M., et al., Review of Upper Limb Hybrid Exoskeletons. IFAC-PapersOnLine, 2017. 50(1): p. 15169-15178.

10. Stewart, A.M., C.G. Pretty, and X. Chen. Design and Testing of a Novel, Low-cost, Low-voltage, Functional Electrical Stimulator. in 2016 12th IEEE/ASME International Conference on Mechatronic and Embedded Systems and Applications (MESA). 2016. IEEE.

11. TheraSigma, Hydrogel Electrodes, in Website. www.therasigma.com.

12. Fleury, A., M. Sugar, and T. Chau, E-textiles in Clinical Rehabilitation: A Scoping Review. Electronics, 2015. 4(1): p. 173-203.

13. Kim, R.H. and G. Cho, Effectiveness of the Smart Healthcare Glove System for Elderly Persons with Hypertension. Human Factors and Ergonomics in Manufacturing \& Service Industries, 2013. 23(3): p. 198-212.

14. Keller, T. and A. Kuhn, Electrodes for Transcutaneous (Surface) Electrical Stimulation. Journal of Automatic Control, 2008. 18: p. 35-45.

15. Transparency Market Research, Electrodes for Medical Devices Market (Diagnostic Electrodes - ECG, EEG, EMG, ERG and Fetal Scalp Electrodes, Therapeutic Electrodes - TENS, Pacemakers, Defibrillators, Electrosurgical and Other Electrodes) - Global Industry Analysis, Size, Share, Growth, Trends and Forecast, 2013 - 2019. 2014. 
16. Sparkfun. Conductive Fabric - 12"x13" MedTex130. 2016 2016]; Available from: https://www.sparkfun.com/products/10070.

17. Sparkfun. Flex Sensor 4.5". 2015; Available from: https://www.sparkfun.com/products/8606.

18. Bergquist, A., et al., Neuromuscular Electrical Stimulation: Implications of the Electrically Evoked Sensory Volley. European Journal of Applied Physiology, 2011. 111(10): p. 2409-2426.

19. Huerta, S.C., et al. A Universal Functional Electrical Stimulator Based on Merged Flyback-SC Circuit. in Power Electronics and Motion Control Conference (EPE/PEMC), 2012 15th International. 2012. IEEE.

20. Cheng, K., et al., Development of a Circuit for Functional Electrical Stimulation. IEEE Transactions on Neural Systems and Rehabilitation Engineering, 2004. 12(1): p. 43-47.

21. Doucet, B.M., A. Lam, and L. Griffin, Neuromuscular Electrical Stimulation for Skeletal Muscle Function. The Yale Journal of Biology and Medicine, 2012. 85(2): p. 201.

22. Agnello, D., New-Generation Fully Programmable Controller for Functional Electrical Stimulation Applications. 2011, University of Toronto.

23. Gorgey, A.S., et al., Effects of Electrical Stimulation Parameters on Fatigue in Skeletal Muscle. Journal of Orthopaedic \& Sports Physical Therapy, 2009. 39(9): p. 684-692.

24. Broderick, B.J., P.P. Breen, and G. ÓLaighin, Electronic Stimulators for Surface Neural Prosthesis. Journal of Automatic Control, 2008. 18(2): p. 25-33.

25. Ferrarin, M. and A. Pedotti, The relationship between electrical stimulus and joint torque: A dynamic model. IEEE transactions on rehabilitation engineering, 2000. 8(3): p. 342-352.

26. Gniotek, K., et al. Innovative Textile Electrodes for Muscles Electrostimulation. in Medical Measurements and Applications Proceedings (MeMeA), 2011 IEEE International Workshop on. 2011. IEEE.

27. Zhou, H., et al., Stimulating the Comfort of Textile Electrodes in Wearable Neuromuscular Electrical Stimulation. Sensors, 2015. 15(7): p. 17241-17257.

28. Weder, M., et al., Embroidered Electrode with Silver/titanium Coating for Long-term ECG Monitoring. Sensors, 2015. 15(1): p. 1750-1759.

29. Lynch, C.L. and M.R. Popovic, Closed-loop control of induced muscle contractions. IEEE Control Systems Megazine, 2008: p. 40-50.

30. Riener, R., J. Quintern, and G. Schmidt, Biomechanical model of the human knee evaluated by neuromuscular stimulation. Journal of Biomechanics, 1996. 29(9): p. 1157-1167.

31. Hussain, Z. and M.O. Tokhi. Modelling of muscle extension and flexion for FES-assisted indoor rowing exercise. in Modeling \& Simulation, 2008. AICMS 08. Second Asia International Conference on. 2008. IEEE.

32. Falisse, A., et al., EMG-driven optimal estimation of subject-specific Hill model muscle-tendon parameters of the knee joint actuators. IEEE Transactions on Biomedical Engineering, 2017. 64(9): p. 2253-2262.

33. Malešević, N.M., et al., A Multi-pad Electrode Based Functional Electrical Stimulation System for Restoration of Grasp. Journal of Neuroengineering and Rehabilitation, 2012. 9(1): p. 1.

34. Schill, O., et al. Automatic Adaptation of a Self-adhesive Multi-electrode Array for Active Wrist Joint Stabilization in Tetraplegic SCI Individuals. in 2009 IEEE Toronto International Conference Science and Technology for Humanity (TIC-STH). 2009. IEEE.

35. Ibitoye, M.O., et al., Strategies for rapid muscle fatigue reduction during FES exercise in individuals with spinal cord injury: a systematic review. PloS one, 2016. 11(2): p. e0149024.

36. Abbas, J.J. and R. Riener, Using mathematical models and advanced control systems techniques to enhance neuroprosthesis function. Neuromodulation: Technology at the Neural Interface, 2001. 4(4): p. 187-195.

37. De Luca, C., Control properties of motor units. Journal of Experimental Biology, 1985. 115(1): p. 125136. 